\title{
Bradyrhizobium diazoefficiens USDA110 PhaR functions for pleiotropic regulation of cellular processes besides PHB accumulation
}

Shogo Nishihata', Takahiko Kondo², Kosei Tanaka², Shu Ishikawa², Shinji Takenaka', Choong-Min Kang ${ }^{3}$ and Ken-ichi Yoshida $2^{2^{*}}$

\begin{abstract}
Background: Bradyrhizobium diazoefficiens USDA110 nodulates soybeans for nitrogen fixation. It accumulates poly3-hydroxybutyrate (PHB), which is of physiological importance as a carbon/energy source for survival during starvation, infection, and nitrogen fixation conditions. PHB accumulation is orchestrated by not only the enzymes for PHB synthesis but also PHB-binding phasin proteins (PhaPs) stabilizing the PHB granules. The transcription factor PhaR controls the phaP genes.

Results: Inactivation of phaR led to decreases in PHB accumulation, less cell yield, increases in exopolysaccharide (EPS) production, some improvement in heat stress tolerance, and slightly better growth under microaerobic conditions. Changes in the transcriptome upon phaR inactivation were analyzed. PhaR appeared to be involved in the repression of various target genes, including some PHB-degrading enzymes and others involved in EPS production. Furthermore, in vitro gel shift analysis demonstrated that PhaR bound to the promoter regions of representative targets. For the phaP1 and phaP4 promoter regions, PhaR-binding sites were determined by DNase I footprinting, allowing us to deduce a consensus sequence for PhaR-binding as TGCRNYGCASMA (R: A or G, Y: C or T, S: C or G, M: A or C). We searched for additional genes associated with a PhaR-binding sequence and found that some genes involved in central carbon metabolism, such as pdhA for pyruvate dehydrogenase and pckA for phosphoenolpyruvate carboxykinase, may be regulated positively and directly by PhaR.
\end{abstract}

Conclusions: These results suggest that PhaR could regulate various genes not only negatively but also positively to coordinate metabolism holistically in response to PHB accumulation.

Keywords: Bradyrhizobium diazoefficiens, Poly-3-hydroxybutyrate (PHB), Transcriptome, Transcription factor, DNA binding

\section{Background}

Poly-3-hydroxybutyrate (PHB) is a type of polyhydroxyalkanoate (PHA), which accumulates within the cells of various microorganisms as an energy storage substance under stress conditions, such as oxygen limitation and nutrient starvation [1]. PHB, a potential substitute for petroleum-based plastics, is produced in an enzymatic

\footnotetext{
* Correspondence: kenyoshi@kobe-u.ac.jp

${ }^{2}$ Department of Science, Technology and Innovation, Kobe University, 1-1

Rokkodai, Nada, Kobe 657 8501, Japan

Full list of author information is available at the end of the article
}

process involving reactions requiring mild conditions and is biodegradable even under opportunistic anaerobic conditions [2].

PHB or PHA accumulation is of physiological importance in many bacterial species. Azospirillum brasilense is a freeliving soil bacterium that affects the growth of numerous agricultural crops [3]. Mutant strains of A. brasilense lacking the ability to accumulate PHA exhibited decreased resistance to various stress conditions, and degradation products of PHB could protect microorganisms from hydroxyl radicals $[4,5]$. In the case of free-living Sinorhizobium

(c) The Author(s). 2018 Open Access This article is distributed under the terms of the Creative Commons Attribution 4.0 International License (http://creativecommons.org/licenses/by/4.0/), which permits unrestricted use, distribution, and 
meliloti, an alfalfa symbiont, PHB is continually synthesized and degraded, which may serve as an energy source under starvation conditions [6]. In addition, a mutant defective in PHB synthesis demonstrated a decreased symbiotic ability and diminished nitrogen fixing ability with weakened nitrogenase activity [7]. In Bradyrhizobium japonicum (Bradyrhizobium diazoefficiens) USDA110, a soybean symbiont bacterium, under the symbiotic state, it was proposed that utilization of PHB might maintain nitrogen fixation activity [8]. In contrast, a mutant of $B$. diazoefficiens USDA110 defective in PHB accumulation had a better symbiotic performance than the wild type [9]. These observations suggest that the physiological importance of $\mathrm{PHB}$ accumulation varies from species to species [10]. Though it has been proposed that PHB is used as an energy source during the bacterial differentiation process from the free-living form to the symbiotic form [11], there remain many unclear points about the physiological significance of PHB.

PHB metabolism in S. meliloti has been elucidated [10]; synthesis of PHB proceeds thusly: first, PhbA (Ketothiolase) synthesizes acetoacetyl-CoA from two molecules of AcetylCoA. Next, PhbB (Acetoacetyl-CoA reductase) reduces Acetoacetyl-CoA to form 3-hydroxybutyryl-CoA. Then, PhbC (PHB synthase) polymerizes 3-hydroxybutyryl-CoA to form PHB. Degradation of PHB proceeds as such: PHB is monomerized by PhaZ (PHB depolymerase) into 3-hydroxybutyrate, which is further oxidized by BdhA (3-hydroxybutyrate dehydrogenase) to produce acetoacetate. Esterification of acetoacetate by AcsA2 (acetoacetylCoA synthetase) produces acetoacetyl-CoA. Finally, acetoacetyl-CoA is decomposed to acetyl-CoA. In $B$. diazoefficiens strain USDA110, which also accumulates PHB in large quantities, PHB metabolism was deduced by genomic comparison with $S$. meliloti, and transcription of the paralogous genes involved in PHB metabolism was analyzed [12]. The genes transcriptionally induced during PHB accumulation in the free-living state were $p h b A 2$ (bll0226), phbB2 (bl10225), phbC3 (bll4360), phbC5 (bll6073), and phaZ1 (blr0908) [12]. In addition, phbC3 notably contributed to PHB synthesis, since its inactivation abolished PHB accumulation [9].

Ralstonia eutropha was studied intensively to elucidate the mechanisms controlling PHB granule stabilization [13], which requires PhaR and phasin proteins (PhaPs) [14]. When the PHB level is low, the PhaR repressor binds to the promoter regions of its own gene and phaP genes to minimize their expression. PhaR binds not only to DNA but also to PHB. Once PHB production is commenced, the intracellular concentration of $\mathrm{PHB}$ reaches a certain level that antagonizes DNA binding of PhaR, and $\mathrm{PhaR}$ dissociates from the promoter regions. At the initial stage of PHB accumulation, PhaR binds to PHB granules instead of DNA, and transcription of the phaP promoters is elevated as PhaR dissociates from DNA to induce PhaP synthesis. Since PhaPs have a higher affinity for PHB than PhaR, the increasing amount of PhaPs induces dissociation of PhaR from PHB. PhaPs are amphipathic and stabilize hydrophobic PHB granules. Finally, PhaR released from PHB binds once again to the promoter regions to repress transcription of the phaR and phaP genes. Repeating this process, PHB accumulates in the cytoplasm as large stabilized granules. In $B$. diazoefficiens USDA110, PHB accumulation is achieved in a similar way as in $R$. eutropha [15]. There are at least four PhaP paralogs and PhaR, which bound to PHB in vitro; PhaP4 exhibited the highest affinity to $\mathrm{PHB}$, which could compete with PhaR to induce its dissociation from PHB [12].

Disruption of phaR in R. eutropha led to a drastic reduction in PHB accumulation [13, 14, 16]. In addition, phaR inactivation in Methylobacterium extorquens resulted in slower proliferation and a decrease in PHB accumulation [17]. Furthermore, under anaerobic conditions, disruption of aniA (phaR) in S. meliloti reduced PHB accumulation but increased the accumulation of exopolysaccharides (EPS) [18]. In addition, aniA (phaR)-disruption in Rhizobium etli [19] and B. diazoefficiens [9] produced similar phenotypes as the $S$. meliloti mutant, even under aerobic conditions. In the present study, we constructed and investigated a phaR-inactive mutant of $B$. diazoefficiens USDA110. Our transcriptomic analysis reveals that PhaR plays an important role not only in PHB accumulation but also in pleiotropic regulation. Furthermore, we analyzed DNA binding of a recombinant PhaR with a C-terminal $\mathrm{His}_{6}$-tag fusion in vitro, and deduced a consensus sequence for PhaR binding, providing a holistic view of PhaR regulon.

\section{Methods}

Bacterial strains, plasmids, culture conditions, and oligonucleotide primers

Bacterial strains and plasmids used in this study are listed in Table 1. Strains of Escherichia coli were cultured in lysogeny broth (LB) [20] at $37^{\circ} \mathrm{C}$ with shaking at $180 \mathrm{rpm}$. Strains of $B$. diazoefficiens were maintained on peptone salts yeast extract medium (PSY) [21] at $28{ }^{\circ} \mathrm{C}$. A fresh colony was selected from a media plate and was inoculated into test tubes containing $5 \mathrm{~mL}$ of PSY and precultured at $28{ }^{\circ} \mathrm{C}$ with shaking. An aliquot of the culture was diluted in $5 \mathrm{~mL}$ of yeast extract mannitol medium (YEM) [22] or tryptone yeast medium (TY) [23] and cultured further at $28{ }^{\circ} \mathrm{C}$ with shaking. When needed, polymyxin $\mathrm{B}(\mathrm{Pm})$ and kanamycin $(\mathrm{Km})$ were added at $50 \mu \mathrm{g} / \mathrm{mL}$, as noted in the following procedures. For cultivation under microaerobic conditions, plates were sealed inside an AnaeroPouch-Anaero anaerobic gas generator system (Mitsubishi Gas Chemical) and incubated at 
Table 1 Bacterial strains and plasmids used in this study

\begin{tabular}{|c|c|c|}
\hline Strains or plasmids & Derivation and relevant properties & Source of reference \\
\hline \multicolumn{3}{|l|}{ B. diazoefficiens } \\
\hline USDA110 & Wild type & [42] \\
\hline$\Delta \mathrm{phaR}$ & A mutant of USDA110 with deletion of phaR & This work \\
\hline \multicolumn{3}{|l|}{ E. coli } \\
\hline $\mathrm{DH} 5 \mathrm{a}$ & supE44 $\Delta$ lacU169 hsdR17 recA1 endA1 gyrA96 thi-1 relA1 & Takara Bio \\
\hline S17-1 & F- thi pro hsdR [RP4-2 tet::Mu kan::Tn7 (trp str)] & [43] \\
\hline BL21(DE3) & $\mathrm{F}^{-}$ompT hadS ${ }_{\beta}\left(\mathrm{r} \beta^{-} \mathrm{m} \beta^{-1}\right) \mathrm{gal} d \mathrm{~cm}(\mathrm{DE} 3)$ & Takara Bio \\
\hline \multicolumn{3}{|l|}{ Plasmids } \\
\hline pK18mobsacB & Mobilizable plasmid containing oriV, oriT, mob, sacB, and kan & [44] \\
\hline pK18mobsacB $\Delta$ phaR & pK18 mobsacB containing the flanking regions of phaR & This work \\
\hline pET28b & kan & Takara Bio \\
\hline pET28PhaR & pET28b carrying the coding region of phaR & This work \\
\hline pMD20 & A linearized vector for TA cloning of PCR fragments & Takara Bio \\
\hline
\end{tabular}

$28{ }^{\circ} \mathrm{C}$. The oligonucleotide primers used in this study are listed in Additional file 1: Table S1.

\section{Mutant construction}

Two DNA fragments were amplified from the chromosomal DNA of $B$. diazoefficiens USDA110 by PCR using the primer pairs FFphaR/FRphaR and RFphaR/RRphaR (Additional file 1: Table S1). The fragments were fused by a recombinant PCR reaction using the primer pairs FFphaR/RRphaR (Additional file 1: Table S1). The fusion PCR product, that corresponds to a mutated phaR with an internal deletion from the 21st to 185 th codons, was trimmed by two restriction enzymes, $\mathrm{XbaI}$ and HindIII, ligated into plasmid $\mathrm{pK} 18 \mathrm{mobsacB}$ (Table 1) cleaved previously with the same enzymes, and transformed into $E$. coli DH5 $\alpha$. Correct construction of the recombinant plasmid was confirmed by DNA sequencing. The plasmid was transferred into E. coli $\mathrm{S} 17-1$, which was then cultured overnight at $37{ }^{\circ} \mathrm{C}$ as the plasmid donor. USDA110 as the recipient was cultured with $\mathrm{Pm}$ at $28{ }^{\circ} \mathrm{C}$ until the $\mathrm{OD}_{600}=1.0$. Equal volumes of the donor and the recipient cultures were mixed and spun down. The collected cells were suspended in sterilized water and pipetted onto a membrane filter (Merck Millipore) placed on a PSY agar plate without antibiotics and incubated overnight at $30{ }^{\circ} \mathrm{C}$. Following this incubation, the bacterial cells on the filter were suspended in sterilized water, spread on PSY agar plates containing both $\mathrm{Pm}$ and $\mathrm{Km}$, and incubated at $28{ }^{\circ} \mathrm{C}$ to form colonies. Chromosomal DNA of some colonies was subjected to PCR using the primers FFphaR and RRphaR to choose a candidate clone having the entire plasmid DNA integrated into the target site of the chromosome. The chosen clone was cultured in PSY containing Pm alone to induce a pop-out event-spontaneous intra-chromosomal recombination of the plasmid
DNA encoding $\mathrm{Km}$ resistance and $s a c B$ genes-and plated on PSY containing both $10 \%$ sucrose and Pm. The resulting colonies were duplicated on two plates, one containing $\mathrm{Km}$ and the other $\mathrm{Pm}$. A clone losing $\mathrm{Km}$ resistance, whose correct construction was confirmed by PCR analysis and DNA sequencing with primers phaR-c-F and phaR-c-R (Additional file 1: Table S1), was designated as strain $\Delta$ phaR with deletion of phaR.

\section{Phenotypic analysis}

To determine PHB accumulation, bacterial cells $\left(1 \mathrm{OD}_{600}\right.$ unit) were collected and suspended in 5\% sodium hypochlorite and incubated overnight at $37{ }^{\circ} \mathrm{C}$. After a brief centrifugation, the precipitate was suspended in distilled water, and mixed with a twofold volume of $99.5 \%$ ethanol and acetone 1:1 mixture. After another spinning down, the precipitant was dissolved in chloroform at $50{ }^{\circ} \mathrm{C}$. The solution was transferred to another tube and air-dried. After addition of $95 \%$ sulfuric acid, the tube was incubated at $100{ }^{\circ} \mathrm{C}$ for $20 \mathrm{~min}$ to lyse the cells, and $\mathrm{OD}_{235}$ of the solution was measured to calculate the amount of PHB. A standard curve was prepared by using various amounts of PHB (Sigma-Aldrich) as previously described [24].

To evaluate EPS production, bacterial cells $\left(1 \mathrm{OD}_{600}\right.$ unit) were spun down, and the supernatant mixed with a threefold volume of ethanol, chilled at $-20{ }^{\circ} \mathrm{C}$ for $10 \mathrm{~min}$, and centrifuged again. The precipitated EPS was air-dried, suspended in water, mixed with $0.2 \%$ anthrone (Nacalai Tesque) in $95 \%$ sulfuric acid, incubated at $100{ }^{\circ} \mathrm{C}$ for $10 \mathrm{~min}$, and cooled under running water. The $\mathrm{OD}_{620}$ of the solution was measured to calculate the amount of EPS as previously described [25].

To measure the intracellular glycogen, bacterial cells were collected and suspended in $30 \% \mathrm{KOH}$ and heated at $100{ }^{\circ} \mathrm{C}$ for $20 \mathrm{~min}$. After adding threefold volume of 
ethanol, the tube was centrifuged and the supernatant discarded. The precipitate was suspended in water, mixed with an equal volume of $0.2 \%$ anthrone in $95 \%$ sulfuric acid, incubated at $100{ }^{\circ} \mathrm{C}$ for $10 \mathrm{~min}$, and cooled under running water. The $\mathrm{OD}_{620}$ of the solution was measured, and a calibration curve was prepared using glucose to calculate the amount of glycogen as described [26].

Stress tolerance was tested as follows: bacterial strains were precultured in TY and YEM to stationary phase. For the heat shock stress experiments, diluted cultures were incubated at $50{ }^{\circ} \mathrm{C}$ for $10 \mathrm{~min}$ with shaking. For the osmotic stress experiments, cells were mixed with glucose, $\mathrm{NaCl}$, or sucrose at various concentrations and then incubated at $28{ }^{\circ} \mathrm{C}$ for $24 \mathrm{~h}$ with shaking. Following incubation, the cultures were serially diluted, and aliquots were spotted on PSY agar plates and incubated at $28^{\circ} \mathrm{C}$.

\section{RNA preparation and analysis}

Strains of $B$. diazoefficiens were grown in $50 \mathrm{~mL}$ of $\mathrm{TY}$ at $28{ }^{\circ} \mathrm{C}$ with shaking until the $\mathrm{OD}_{600}=0.4$. Cells from these cultures were harvested and suspended in $1 \mathrm{~mL}$ of LETS buffer $(100 \mathrm{mM} \mathrm{LiCl}, 10 \mathrm{mM}$ EDTA, $10 \mathrm{mM}$ Tris- $\mathrm{HCl}$ at $\mathrm{pH} 7.4$ ), vortex mixed with $0.5 \mathrm{~mL}$ of glass beads $(\phi 0.5 \mathrm{~mm})$ and $1 \mathrm{~mL}$ of phenol for $6 \mathrm{~min}$, and centrifuged. After centrifugation, $1 \mathrm{~mL}$ of the upper aqueous layer was mixed with $1 \mathrm{~mL}$ of phenol-chloroform for $30 \mathrm{~s}$. After another centrifugation, $0.75 \mathrm{~mL}$ of the upper aqueous layer was mixed with $0.1 \mathrm{~mL}$ of $1 \mathrm{M} \mathrm{LiCl}$ and $2.5 \mathrm{~mL}$ of ethanol and centrifuged. The supernatant was discarded, and the precipitate was dissolved in $0.175 \mathrm{~mL}$ of distilled water, mixed with 3.5-fold volume of $4 \mathrm{M}$ sodium acetate $(\mathrm{pH} 6.0)$, incubated at $-20{ }^{\circ} \mathrm{C}$ for a minimum of $1 \mathrm{~h}$, and centrifuged. The RNA precipitate was dissolved in distilled water, and treated with DNase I at $37{ }^{\circ} \mathrm{C}$ for $1 \mathrm{~h}$. Then DNA-free RNA was purified using RNeasy Mini Kit (Qiagen). Concentrations of the purified RNA samples were determined with Nano Vue Plus (GE Healthcare), and the samples were stored in aliquots at $-80{ }^{\circ} \mathrm{C}$ prior to the usage.

The mRNA transcription start points were determined by the $5^{\prime}$-RACE method using the $5^{\prime}$-Full RACE Core Set (Takara Bio). 50 ng of RNA was used as the template for reverse transcription, producing the first cDNA strand from the specific primers, RT-primer phaP1-phos and RT-primer phaP4-phos (Additional file 1: Table S1). After treatment with RNase $\mathrm{H}$, the resulting single stranded cDNA was incubated with T4 RNA ligase overnight at $15{ }^{\circ} \mathrm{C}$. The ligated cDNA served as the template in the subsequent nested PCRs with the following primer pairs: phaP1S1/phaP1A1 followed by phaP1S2/phaP1A2 for phaP1; and phaP4S1/phaP4A1 followed by phaP4S2/ phaP4A2 for phaP4 (Additional file 1: Table S1). The resulting PCR fragment was cloned into a pMD20 (Takara Bio) and sequenced to identify the transcription initiation point.
For quantitative RT-PCR, 10 ng of RNA was used as a template for reverse transcription using a ReverTraAce qPCR RT Kit (Toyobo) and each of the reverse primers (Additional file 1: Table S1; RT primer pairs were systematically designated beginning with "RT-," followed by the respective gene name, and "-F" or "- $R$ " at the end). Reverse transcription products were subjected to relative quantification PCR using Thunderbird Sybr qPCR Mix (Toyobo) and the specific primer pairs (Additional file 1: Table S1) in a Thermal Cycler Dice Real Time System MRQ (Takara Bio).

For RNA-sequencing transcriptome analysis, the total RNA was treated using a Ribo-Zero rRNA Removal Kit (Bacteria) (Illumina) to eliminate rRNA, treated with an Agilent RNA Pico kit (Agilent Technologies), and analyzed for purity using a 2100 Bioanalyzer (Agilent Technologies). The mRNA was converted into a cDNA library by using a NEBNext Ultra Directional RNA Library Prep Kit for Illumina (New England Biolabs), NEB Next Multiplex Oligos for Illumina Index Primers Set I (New England Biolabs), and an Agencourt AMPure XP PCR purification system (Beckman Coulter). Quality of the cDNA library was evaluated using an Agilent High Sensitivity DNA Kit and 2100 Bioanalyzer (Agilent Technologies). The cDNA library was subjected to sequencing using a MiSeq Reagent kit $\mathrm{v} 3$ (150 cycles) (Illumina) in Illumina MiSeq (Illumina).

\section{Databases and software}

RNA sequencing data were analyzed using CLC Genomics Workbench version 6.5.1 (Qiagen) as follows. The complete genome sequence of $B$. japonicum (diazoefficiens) USDA110 was obtained as a reference from the NCBI database (accession number NC_004463.1). Quality-filtered sequencing reads were aligned to the reference genome sequence. Mapping was based on a minimal length of $100 \mathrm{bp}$ with an allowance of up to two mismatches. Relative transcript abundance was measured in reads per kilobase per megabase of library size (RPKM). Results of the analysis were submitted to the DDBJ Sequence Read Archive (DRA) database under accession number DRA005621. Gene functions were predicted using the Kyoto Encyclopedia of Genes and Genomes (http://www.genome.jp/kegg/) and rhizobase (http://genome.microbedb.jp/rhizobase/). PhaR-binding sequences were predicted by GLAM2 version 1056 (http://meme-suite.org/tools/glam2) in MEME suite version 4.11.2 (http://meme-suite.org/) to detect a motif for consensus sequence under the default settings.

\section{Preparation of PhaR-His}

B. diazoefficiens phaR was expressed in E. coli BL21(DE3) to prepare PhaR with a C-terminal $\mathrm{His}_{6}$-tag (PhaR-His 6 ) as follows: a PCR fragment of the phaR-coding region was amplified from the DNA of USDA110 using the primer pair pET28b-phaR-gib-F/pET28b-phaR-gib-His6-R 
(Additional file 1: Table S1). Another PCR fragment was made from plasmid pET28b (Table 1, Takara Bio) with the primer pair pET28b-inverse NcoI-F/pET28b-inverse BamHI-R (Additional file 1: Table S1). This fragment was ligated with the phaR fragment using a Gibbson assembly kit (New England Biolabs) to obtain the plasmid pET28PhaR (Table 1), the correct construction of which was confirmed by sequencing. pET28PhaR was introduced into BL21(DE3), which was cultured in LB containing $\mathrm{Km}$ until the $\mathrm{OD}_{600}$ reached 0.6 , and phaR was induced by the addition of $1 \mathrm{mM}$ IPTG for $4 \mathrm{~h}$. The cells were harvested and suspended in a buffer $(50 \mathrm{mM}$ phosphate at $\mathrm{pH}$ 8.0, $20 \%$ glycerol, and $0.5 \mathrm{M} \mathrm{NaCl}$ ), and disrupted by sonication in an ice bath. From the lysate, $\mathrm{PhaR}^{-\mathrm{His}_{6}}$ was purified using TALON Metal Affinity Resin (Takara Bio).

\section{Gel shift assay and DNase I footprinting}

DNA fragments used for the gel shift assay were PCR fragments amplified from the DNA of USDA110 using the specific primer pairs (Additional file 1: Table S1; They were systematically designated beginning with "EMSA-", followed by the respective gene name, and "-F" or "-R" at the end). The DNA fragments were combined in the binding-reaction mixture containing $285 \mathrm{mM}$ Tris $-\mathrm{HCl}(\mathrm{pH}$ 8.0), $0.14 \mathrm{mg} / \mathrm{mL}$ bovine serum albumin, $1.43 \mathrm{mM}$ dithiothreitol, $5 \%$ glycerol, $0.04 \mathrm{mg} / \mathrm{mL}$ poly-deoxyinosinic-deoxycytidylic acid (Merck), and $3.06 \mathrm{mM}$ EDTA. Addition of serially diluted PhaR-His ${ }_{6}$ yielded a total reaction volume of $10 \mu \mathrm{L}$, which was incubated for $30 \mathrm{~min}$ at $25{ }^{\circ} \mathrm{C}$. The reaction was then mixed with $80 \%$ glycerol and applied to 6\% PAGE in TAE buffer $(\mathrm{pH}$ 8.2) at $100 \mathrm{~V}$ and $20 \mathrm{~mA}$ for $2 \mathrm{~h}$. The gel was stained with SYBR Green, and the DNA was visualized using the Gel Doc XR+ system (Bio-Rad).

For DNase I footprinting analysis, DNA fragments were differentially labeled at the $5^{\prime}$-teminus on either strand of DNA using PCR with FAM-labeled primers (Additional file 1: Table S1, the primers designated beginning with FAM were labeled at 5 '-end). For labeling the upper and lower strands of the phaP1 promoter region fragment, the primer pairs FAM-phaP1-F/EMSA-phaP1-R and EMSA-phaP1-F/FAM-phaP1-R were used, respectively. For labeling the upper and lower strands of the phaP4 promoter region fragment, the pairs of FAM-phaP4-F/EMSA-phaP4-R and EMSA-phaP4-F/FAM-phaP4-R were used, respectively. Various amounts of purified $\mathrm{PhaR}-\mathrm{His}_{6}$ and each DNA fragment were combined in the binding reaction and incubated for $30 \mathrm{~min}$ at $25{ }^{\circ} \mathrm{C}$. Subsequently, the mixture was treated with $0.025-0.1 \mathrm{U} / \mu \mathrm{l}$ DNase I (Takara Bio) for $5 \mathrm{~min}$. The reaction was terminated by the addition of EDTA, and the DNA in the mixture was purified using a QIAquick Nucleotide Removal Kit
(Qiagen). Corresponding sequence ladders were prepared using a Thermo Sequenase Dye Primer Manual Cycle Sequencing Kit (Thermo Fisher Scientific) according to the standard procedure. The fragment analysis was outsourced to Sigma-Aldrich, Japan.

\section{Results}

\section{Phenotypic changes caused by inactivation of phaR}

Two strains of $B$. diazoefficiens, the wild-type USDA110 and its mutant $\triangle$ phaR, were grown in TY and YEM (Fig. 1a and b, respectively). TY is rich in nitrogen sources, and USDA110 grown in TY does not accumulate PHB [12]. When grown in TY, there were no obvious differences in the growth of the two strains in 7 days (Fig. 1a). In YEM, which has a higher carbon/nitrogen ratio, USDA110 is known to accumulate PHB [12]. USDA110 was able to grow to an $\mathrm{OD}_{600}>1.0$ in 20 days in YEM (Fig. 1b), and its PHB content gradually accumulated up to $140 \mu \mathrm{g} / \mathrm{OD}_{600}$ in 12 days (Fig. 1c). In contrast, $\triangle$ phaR grew in YEM with less cell yield with an $\mathrm{OD}_{600} \leq 0.5$ in 12 days (Fig. 1b), and its PHB accumulation was observed at the highest level of $35 \mu \mathrm{g} / \mathrm{OD}_{600}$ after 4 days, but decreased later to leave only residual amounts after 12 days (Fig. 1c). USDA110 produces EPS when grown in YEM, and its EPS production was observed up to $12 \mu \mathrm{g} / \mathrm{OD}_{600}$ in 4 days, and decreased to about $5 \mu \mathrm{g} /$ $\mathrm{OD}_{600}$ after 12 days. The EPS production of $\Delta$ phaR was nearly constant, around $10 \mu \mathrm{g} / \mathrm{OD}_{600}$ during the 12 day growth (Fig. 1d). Both USDA110 and $\Delta$ phaR produced similar intracellular glycogen, at $11.1 \pm 1.14$ and $11.7 \pm$ $2.32 \mu \mathrm{g} / \mathrm{OD}_{600}$ in 12 days, respectively (data not shown).

The defects in phaR led to changes in stress tolerance. After incubation at $50{ }^{\circ} \mathrm{C}, \Delta \mathrm{phaR}$ demonstrated better survival than USDA110 on both TY and YEM plates (Additional file 2: Figure S1). In contrast, under the osmotic stress conditions with elevated concentrations of $\mathrm{NaCl}$, sucrose, or glucose, $\Delta \mathrm{phaR}$ grew similarly to USDA110 (data not shown). Surprisingly, when cultured on TY agar plates under microaerobic conditions, $\Delta$ phaR grew better than USDA110, whereas the two strains exhibited no difference in growth under aerobic conditions (Additional file 3: Figure S2).

Taken together, inactivation of phaR caused various changes in the phenotype of $B$. diazoefficiens, including reduced growth and decreased PHB accumulation, along with elevated production of EPS when grown in YEM. In addition, it enhanced tolerance to heat-shock and microaerobic stresses. These results imply that phaR may be involved in regulation of not only PHB accumulation but also pleiotropic cellular functions.

Transcriptomic changes caused by inactivation of phaR B. diazoefficiens USDA110 does not accumulate PHB in $\mathrm{TY}$, and thus we can assume that in these growth 

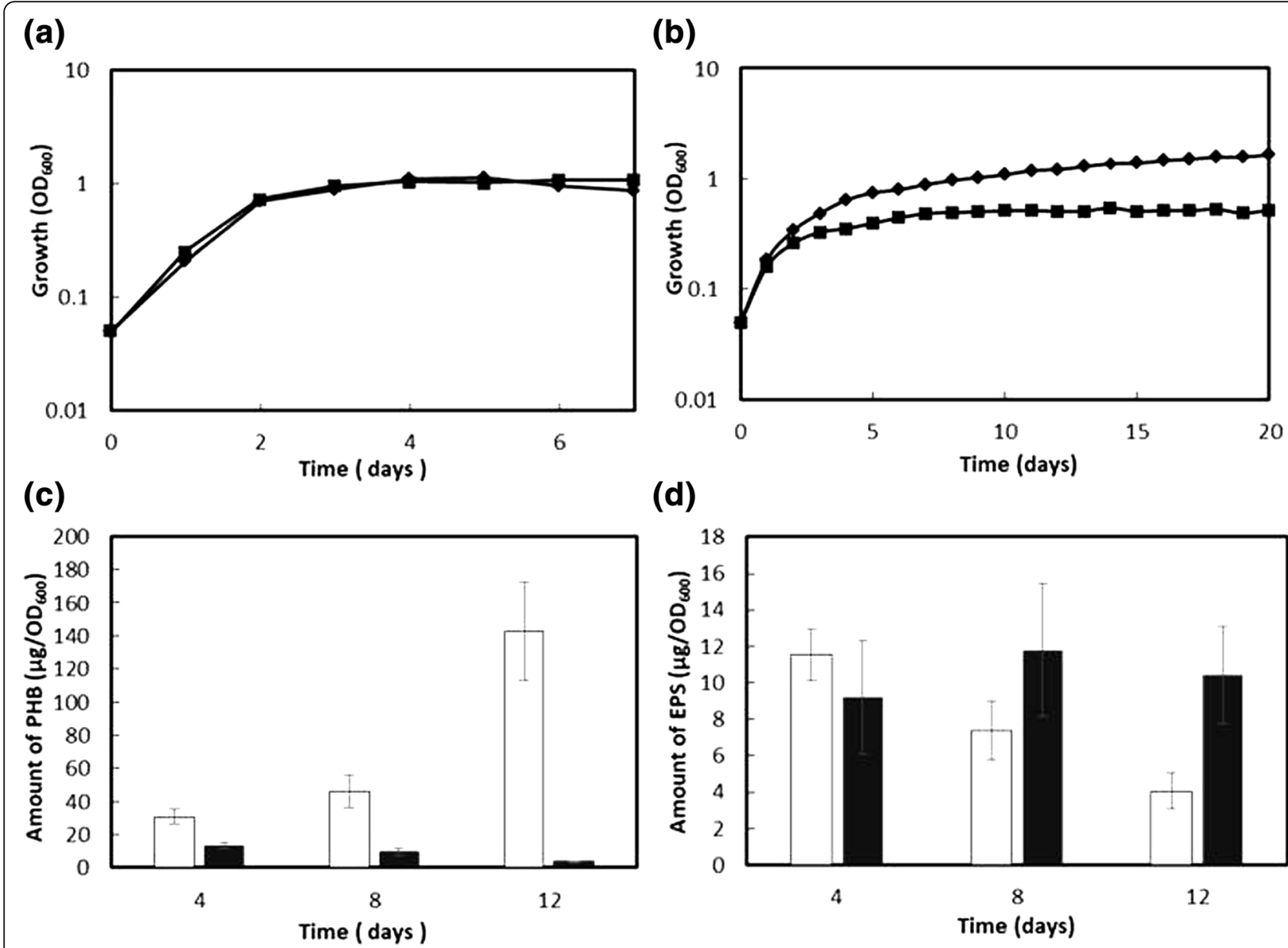

(d)

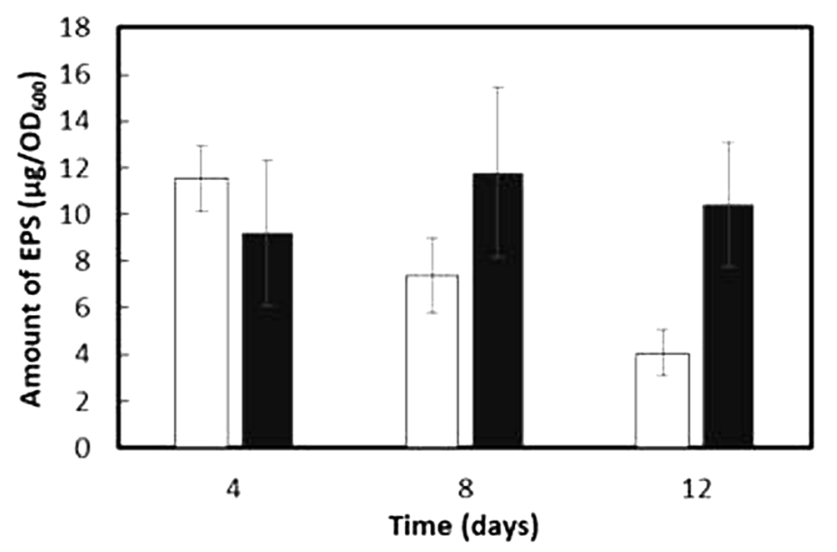

Fig. 1 Physiological characterization of $\Delta$ phaR. a and $\mathbf{b}$ Growth curves of strains USDA110 (solid diamonds) and $\Delta$ phaR (solid squares) of $B$. diazoefficiens grown in TY (a) and YEM (b). A set of representative results from three independent experiments is shown. $\mathbf{c}$ PHB accumulation in USDA110 (open bar) and $\triangle$ phaR (solid bar) grown in YEM. Values are means \pm standard deviation of three independent experiments. $\mathbf{d}$ EPS production in USDA110 (open bar) and $\Delta$ phaR (solid bar) grown in YEM

conditions PhaR exerts its regulatory function to repress phaP genes, as PHB granules are not developing [12]. To test this possibility, USDA110 and $\Delta$ phaR were grown in TY, and total RNA was extracted and subjected to analyze transcriptomic changes.

Quantitative RT-PCR analysis was performed as previously reported [12]. First, we analyzed the expression of the four phaP paralogs, including phaP1 (open reading frame bl15155), phaP2 (bl1555), phaP3 (bl16129), and phaP4 (bl7395). As shown in (Fig. 2a), transcription of phaP1 and phaP4 increased in $\triangle \mathrm{phaR}$ compared with that in USDA110. In contrast, transcription of both phaP2 and phaP3 remained low and did not change. Next, we analyzed the expression of the other eight genes involved in PHB synthesis, phbA1 (blr3724), phbA2 (bll0226), phbB1 (bll3725), phbB2 (bll0225), phbC1 (blr2885), phbC2 (blr3732), phbC3 (bll4360), phbC4 (bll4548), and phbC5 (bll6073), as well as the two genes involved in PHB degradation, phaZ1 (blr0908) and phaZ2 (blr6703) (Fig. 2b).
Expression of phbA1, phbB1, phbC1, phbC2, phbC3, and $p h b C 4$ remained at relatively low levels, whereas expression of $p h b A 2$ remained constitutive without obvious change. Interestingly, $\triangle \mathrm{phaR}$ demonstrated an approximately twofold decrease in $p h b B 2$ expression compared with that in USDA110, whereas expression of both $p h b C 5$ and $p h b C 3$ was elevated. With respect to the PHB-degrading genes, transcription of phaZ1 was elevated in $\triangle$ phaR almost threefold over that of USDA110, whereas expression of phaZ2 was only negligible. The quantitative RT-PCR experiments revealed that PhaR could be involved in regulation of not only phaP1 and phaP4 but also phbB2 and phaZ1 involved in PHB synthesis and degradation, respectively. In addition, inactivation of $p h a R$ led to various changes in phenotype, as described above. These results demonstrate that PhaR may regulate a large number of targets.

In order to assess the possibility, a transcriptome analysis as a screening test was performed by means of 

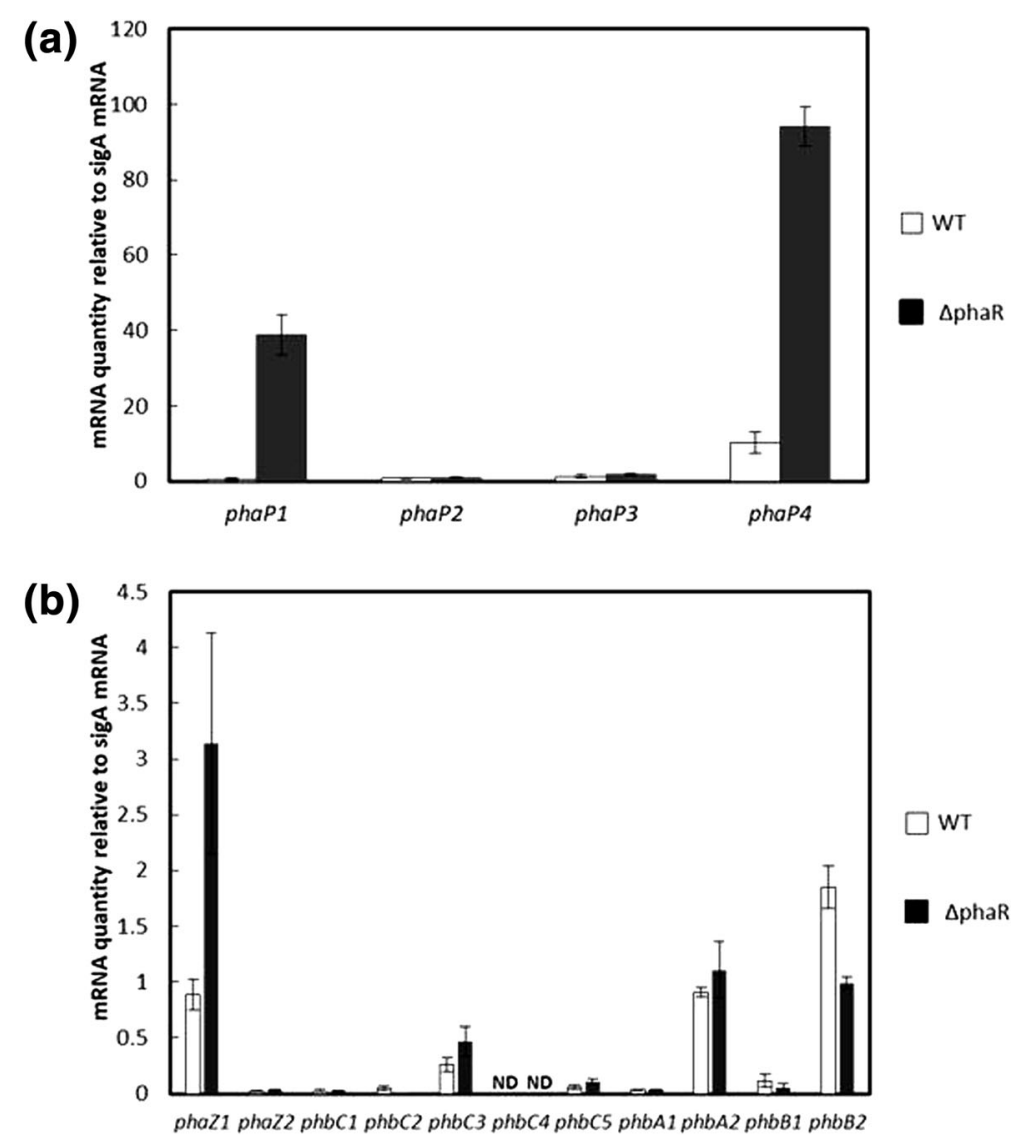

Fig. 2 Transcription levels of phaP paralogs (a) and phbA, phbB, phbC, and phaZ paralogs (b) in USDA110 (WT, open bar) and $\Delta$ phaR (solid bar) grown in TY. Quantitative RT-PCR was performed as described in the main text to determine the transcription levels of the respective genes indicated along the horizontal axis, and data were normalized to the constitutive expression of sigA as the housekeeping sigma factor. Values are means \pm standard deviation of three biologically independent experiments. ND: Not detected

RNA sequencing on the RNA samples prepared from the cells grown in TY (Additional file 4: Table S2). As shown (Additional file 5: Figure S3), the distribution of RPKM values indicated that some genes exhibited altered expression with phaR inactivation. The genes in $\Delta \mathrm{phaR}$ found to be induced in quantitative RT-PCR (Fig. 2) were also induced in RNA sequencing (Additional file 5: Figure S3). Nevertheless, RNA sequencing was more sensitive than quantitative RT-PCR, and it suggested that expression of 77 genes was elevated for more than fourfold in $\triangle \mathrm{phaR}$ (Additional file 4: Table S2). Expression of phaP1, phaP4, and phaZ1 was greatly increased in $\triangle \mathrm{phaR}$ as compared with USDA110. We found that the transcription of phaP5 (blr2887), which may encode an additional PhaP, prominently increased in $\triangle \mathrm{phaR}$. In addition, there was an elevation in the expression of the $c y o A B C D$ operon, which encodes the cytochrome $o$ ubiquinol oxidase complex, one of the terminal oxidase complexes of the electron transport chain, which is usually induced in adaptation to microaerobic conditions [27]. Expression of the operon blr2367-blr2371 containing exoZ, encoding an acetyltransferase that transfers an acetyl group to EPS, was also increased, implying that this operon may be involved in the retained synthesis of EPS in $\triangle$ phaR (Fig. 1d). In $\triangle \mathrm{phaR}$, there were increases in the expression of fixNOQP (cbb3-type cytochrome $c$ oxidase), fixGHIS (Fe-S-binding protein), hemN2 (coproporphyrinogen III oxidase), ppsA (phosphoenolpyruvate synthase), and $n n r R$ (regulator for nitric oxide metabolism), which are regulated by FixK $_{2}$ [28]. In addition, the napEDABC operon (periplasmic nitrate reductase system) and nirK (copper-containing nitrite reductase) were also induced, which are reported to be induced by NnrR during adaptation to anaerobic conditions $[29,30]$. These results suggest that inactivation of $p h a R$ may activate the FixK $K_{2}$ and NnrR regulons. Furthermore, some stress response genes also experienced increased transcription, including blr2761 and bll6069, whose products contain the Usp (universal stress protein) motif [31].

Unexpectedly, a large number of genes were suggested to decrease transcription in $\Delta$ phaR compared with USDA110 (Additional file 4: Table S2), as expression of 119 genes was 
lowered for more than fourfold in $\Delta \mathrm{phaR}$, including a putative operon for $\mathrm{ABC}$ transporter (blr0308-blr0312), a large gene cluster for polyketide synthesis including another $\mathrm{ABC}$ transporter (bll3369-bll3384), and the other genes related to flagellar formation and movement, such as $f l i F 1, f l i R 2$, flhA2, flhB2, flgB2, flgC2, flgE2, flgG2, flgL2, motB2, and motC. The results further suggest that PhaR may upregulate some active transport systems as well as cellular motility.

\section{DNA binding of PhaR}

Gel mobility shift assays using serially concentrated PhaR-His 6 produced and purified in E. coli (Additional file 6: Figure S4) revealed that $\mathrm{PhaR}-\mathrm{His}_{6}$ bound to the promoter region of phaP1, phaP4, phaP5, and phaR itself (Fig. 3a-d). Of the four DNA fragments, PhaR-His 6 bound to the phaP1 promoter most efficiently, as a decrease in the DNA fragment band was observed at $6.89 \mathrm{nM}$ of PhaR-His ${ }_{6}$, and a distinct DNA-protein complex band appeared at $55 \mathrm{nM}$ (Fig. 3a). However, for the phaP4 promoter fragment, shifting of the band occurred at $13.8 \mathrm{nM}$ and the complex appeared at $110 \mathrm{nM}$ (Fig. 3b). For the fragment containing the phaP5 promoter, which was suggested as an additional PhaR target after the transcriptome analysis described above, shifting of the band occurred at $27.8 \mathrm{nM}$ and the complex was formed at $110 \mathrm{nM}$ (Fig. 3c). These results indicated that PhaR-His 6 bound to the promoter regions of phaP1, phaP4, and phaP5. In addition, PhaR-His 6 was shown to bind to the DNA fragment of the phaR promoter (Fig. 3d), but only half of the DNA formed the complex at the highest

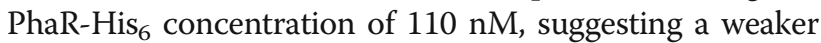
affinity for the phaR promoter region. Next, gel mobility shift assays were performed as stated above with DNA fragments from the phaZ1 and phaZ3 (blr0899) promoters for PHB degrading enzymes (Fig. 3e and f, respectively). The phaZ1 fragment shifted at $27.8 \mathrm{nM}$ and completed the complex formation at $110 \mathrm{nM}$ (Fig. 3e), whereas the phaZ3 fragment shifted at $27.8 \mathrm{nM}$ and completed the complex at $55 \mathrm{nM}$ (Fig. 3f). These results suggested that these genes for PHB degrading enzymes could be regulated directly by PhaR.

Further, we assessed $\mathrm{PhaR}_{-}-\mathrm{His}_{6}$ binding to the promoter regions of the possible PhaR targets suggested by the RNA sequencing transcriptome analysis (Fig. $3 g$ and h). The DNA fragment from the promoter region of $c y o A$ (cyoABCD operon) shifted at $27.8 \mathrm{nM}$ of PhaR-His 6 and completed the complex formation at $55 \mathrm{nM}$, suggesting higher affinity (Fig. 3g). The fragment from the promoter region of blr2367 for the exoZ-containing operon, shifted at $27.8 \mathrm{nM}$ and completed the complex at $110 \mathrm{nM}$ (Fig. 3h). In contrast, despite that its transcription was elevated more than fourfold in $\triangle$ phaR, no PhaR-His ${ }_{6}$ binding to the fragments containing promoter regions of fixN, ppsA, nnrR, and hemN2 was detected, suggesting that PhaR may not directly regulate the expression of these genes (data not shown).

We performed additional gel mobility shift assays on the promoter regions of phaP1 and phaP4 in the presence of PHB (Additional file 7: Figure S5). In both cases, the PhaR-DNA complex formation decreased as the concentration of PHB increased. When $50 \mathrm{ng}$ of $\mathrm{PHB}$ was added to the reaction mixture, binding of $\mathrm{PhaR}^{-\mathrm{His}_{6}}$ to the DNA was almost abolished, as the DNA bands appeared at the same position as the control without PHB, suggesting that PHB could inactivate DNA binding of PhaR-His 6 .

In order to determine the PhaR-His 6 binding sites, we performed DNase I footprint analyses on the phaP1 and phaP4 promoter regions (Fig. $4 \mathrm{a}$ and b, respectively). We found two sites occupied by $\mathrm{PhaR}^{-\mathrm{His}_{6}}$ within the phaP1 promoter region and one in the phaP4 promoter region. In addition, the respective transcription start points $(+1)$ for the two promoters were determined by the 5 '-RACE method using the RNA samples prepared from the $\triangle \mathrm{phaR}$ cells grown in TY, and the corresponding -10 and -35 regions were deduced (Fig. 4). The two PhaRbinding sites with 12-mer sequences TGCGACGCACAA and TGCGTCGCACAA in the phaP1 promoter region are located 6 bp upstream and downstream of the -35 region, respectively, and the binding site with a sequence TGCG CTGCACAA in the phaP4 promoter region overlaps the 35 region. The presence of two binding sites within the phaP1 promoter region may be responsible for its higher affinity to $\mathrm{PhaR}^{-\mathrm{His}_{6}}$ in vitro.

\section{Additional genes regulated by PhaR}

Since PhaR-His 6 bound to the DNA fragments containing the additional six promoter regions, including those of phaP5, phaR, phaZ1, phaZ3, the cyoABCD operon, and the exoZ-containing operon starting with blr2367 (Fig. 3), we speculated that these promoter regions could share a sequence similarity. Nucleotide sequences of the six promoter regions were aligned with three 12-mer sequences of the PhaR-binding sites found in the phaP1 and phaP4 promoter regions (Fig. 4) by GLAM2 in MEME suit to deduce a motif of consensus sequence for PhaR binding as TGCRNYGCASMA (R: A or G, Y: C or T, S: C or G, M: A or C) (Fig. 5).

We searched for sequences similar to the consensus sequence within the putative promoter regions of the genes whose transcription increased or decreased more than twofold in $\Delta$ phaR compared with USDA110 (Additional file 4: Table S2). Their putative promoter regions were selected on the basis of the possible transcription start points reported in recent studies [32]. We extracted 28 and 42 genes associated with the PhaR-binding consensus-like sequence, whose transcription increased (Table 2) and decreased 

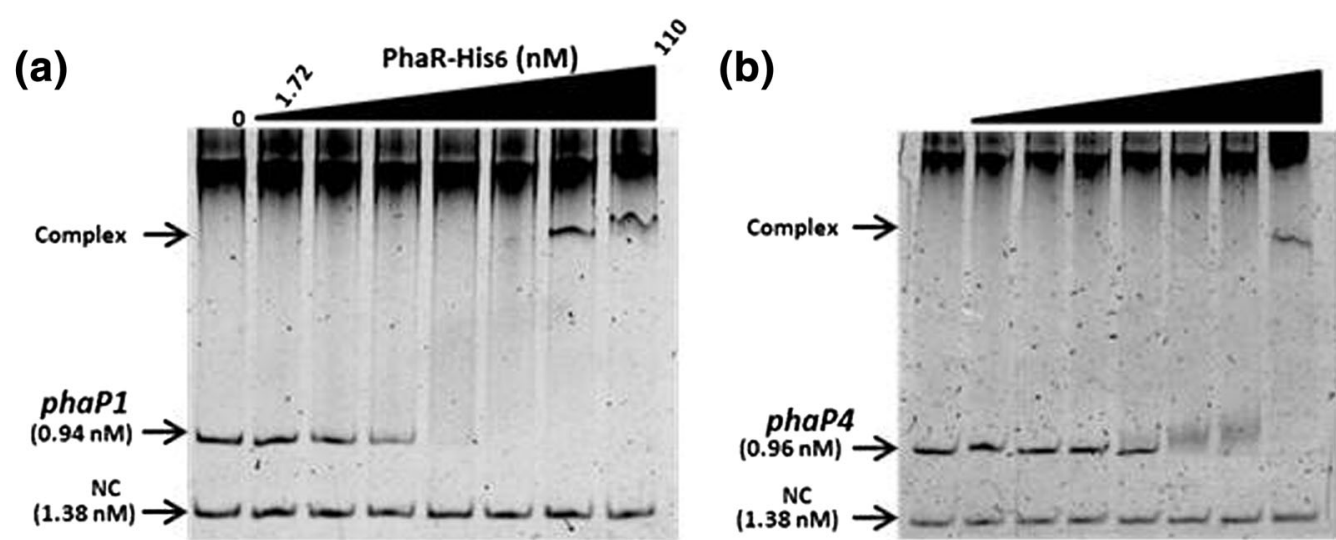

(c)

(d)
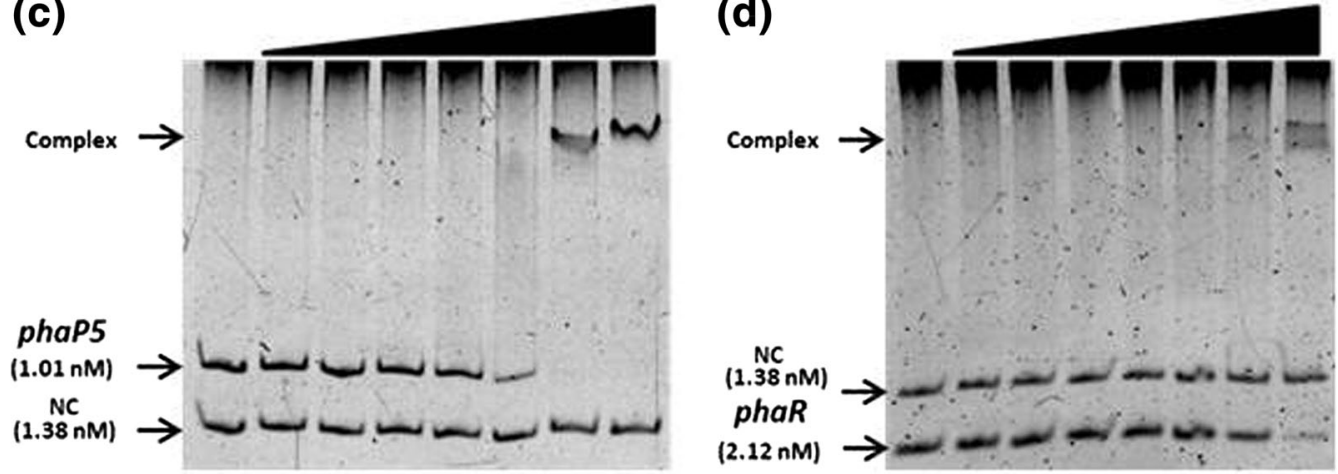

(e)

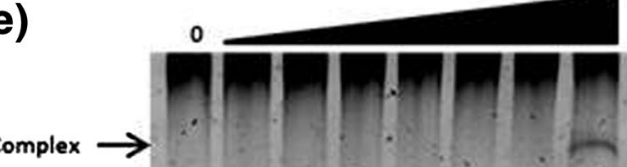

(f)

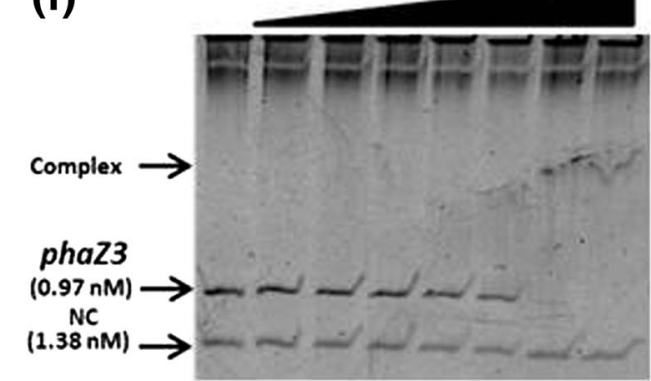

(g)

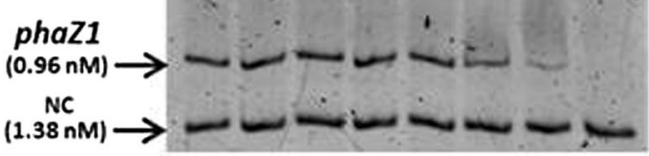

$(0.97 \mathrm{nM}) \rightarrow \ldots \ldots$

$\mathrm{NC}$

(h)
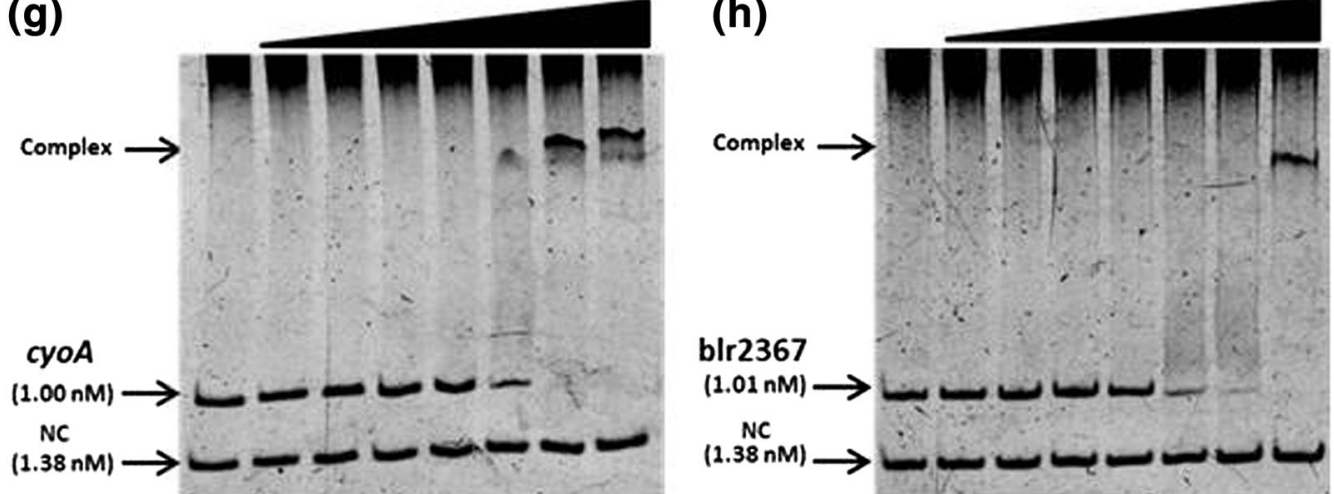

Fig. 3 (See legend on next page.) 
(See figure on previous page.)

Fig. 3 PhaR binding to DNA fragments containing the promoter regions of phaP paralogs (a-c), phaR (d), phaZ paralogs (e and f), and others ( $g$ and $h$ ). Various amounts of purified PhaR-His 6 were incubated with a fixed amount of DNA fragments containing the promoter regions as indicated; (a) phaP1 (bll5155), (b) phaP4 (bll7395), (c) phaP5 (blr2887), and (d) phaR (blr0227), (e) phaZ1 (blr0908), (f) phaZ3 (blr0899), (g) cyoA (blr0149), and (h) blr2367. The reactions were subjected to gel mobility shift assays as described in the main text. Each of the gels has eight lanes containing serially increased amounts of PhaR-His ${ }_{6}$ from left to right to give $0,1.72,3.44,6.88,13.8,27.5$, 55, and 110 nM, respectively. Positions for DNA-protein complex and free DNA fragments are indicated with arrowheads on the left side of the panels. As a negative control (NC), a constant amount of the DNA fragment corresponding to part of the phaP1 coding region is included, which is another PCR fragment amplified using the primer pairs EMSA-phaP1-ORF-F/EMSA-phaP1-ORF-R (Additional file 1: Table S1)

(Table 3) in $\Delta$ phaR, respectively. Among the genes whose transcription was increased in $\Delta$ phaR (i.e., PhaR-repressed), the $p p c$ (encoding phosphoenolpyruvate carboxylase) promoter region demonstrated a weaker affinity for PhaR-His ${ }_{6}$, as approximately half of the DNA band shifted only at the highest PhaR-His ${ }_{6}$ concentration of $110 \mathrm{nM}$ (Fig. 6). However, among the genes whose transcription decreased (PhaR-activated), DNA fragments containing the promoter regions of bll5961 (encoding a putative regulatory protein of Crp family), exaA (quinoprotein ethanol dehydrogenase), $p c k A$ (phosphoenolpyruvate carboxykinase), $p d h A$ (pyruvate dehydrogenase subunit alpha), and phbB2 demonstrated some mobility shift at $110 \mathrm{nM}$ of PhaR-His ${ }_{6}$ (Fig. 6).
The results suggested that these consensus-sequence sites might have lower affinities to PhaR but possibly be involved in the regulation by PhaR.

\section{Discussion}

In the present study, phenotypic changes resulting from phaR inactivation were investigated. There were no observable differences between $\Delta \mathrm{phaR}$ and USDA110 grown in TY, whereas $\Delta$ phaR demonstrated decreased cell yield and diminished PHB accumulation in YEM (Fig. 1). These results suggest that altered $\mathrm{PHB}$ metabolism compromises the balance in intracellular carbon source usage. In fact, expression of $p d h A B$ for acetyl-CoA synthesis was reduced (a)

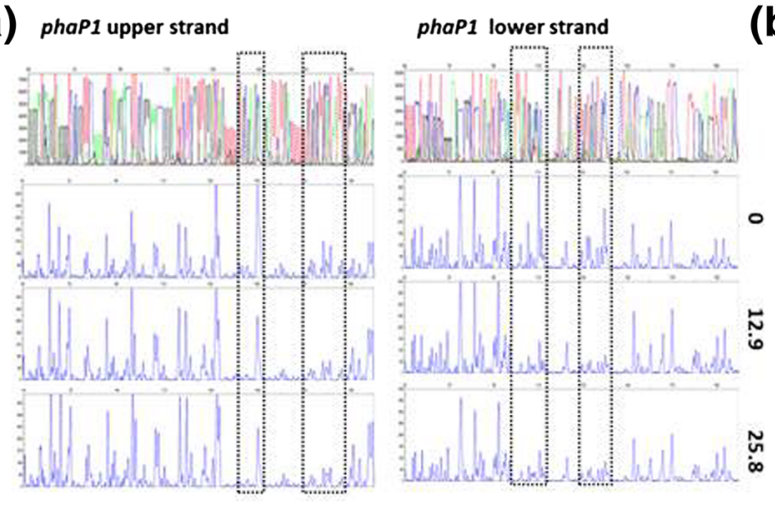

GgtTTCCAAAAGCTTAAGGAACCCTTGCGgGAAACCCCGgGAATCTCTTCGgItTGGA CCAAAGG ITT TCGAATTCCTTGGGAACGCCCTTTGGGGCCCTTAGAGAAGCCAAACCT

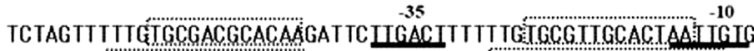
AGATCAAAAACACGCTGCG TGTITCTAAGAACTGAAAAAACACGCAACG TGATTAAEAC +1 队

GGCAGAGTCAGGGAGCCGgGGCTTCCCGACGCGAGCCAGGgAAAAGGATTCCGAAATO CCGTCTCAGTCCCTCGGCCCCGAAGGGC TGCGC TCGGTCCCTTTTCCTAAGGCTTTAC (b)
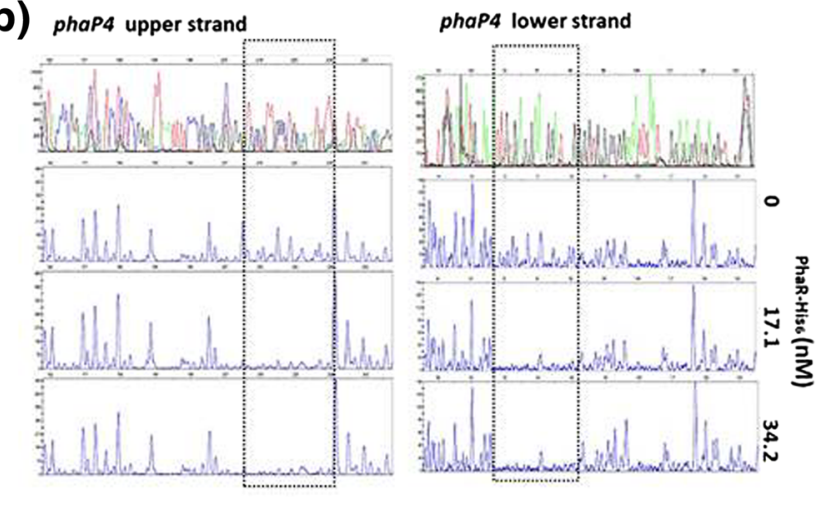

CGgGTTCCCGTACCCCGgaACCTCATCCTCTCCGGGATTAAATTTTCCCGGCGCACCC GCCCAAGGGCATGGGGCCTTGGAGTAGGAGAGGCCCTAATTTAAAAGGGGGCGTGGG

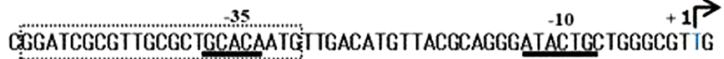
GECTAGCGCAACGCGACG TGT TACAACTGTACAATGCGTCCC TATGACGACCCGCAAC

GACGAGCCATCTIIITCGTTACGCGTATCCAGTCTTCGTACCCGCCAACAGATGGCCC CIGCTCGGTAGAAAAAGCAATGCGCATAGGTCAGAAGCATGGGCGGTTGTCTACCGGG

\section{CGGTIGGCCGGCGGgCGCGCCAGAAGGCTCACTCCATITICCATGATCGTGAGGGACA GCCAACCGGCCGCCCGCGCGGTCTTCCGAGTGAGGTAAAAGGTACTAGCACTCCCTGT \\ ACAATG \\ TGTTAC}

Fig. 4 DNase I footprints of PhaR on the phaP1 (a) and phaP4 (b) promoter regions. DNasel footprints of PhaR-His 6 binding found in DNA fragment patterns are shown for the PhaP1 (a) and phaP4 (b) promoter regions; the fragment patterns on upper and lower strands are in the left and right side of each panel, respectively. Each of the panels contain four fragment charts for respective upper and lower strands; from the top to down, the first is the sequencing ladders in four colors, the second is the negative control without PhaR-His ${ }_{6}$, and the third and fourth are the two different concentrations of PhaR-His 6 as indicated. At the bottom of each panel, nucleotide sequences of the promoter regions of phaP1 (a) and phaP4 (b) are shown. The sequence stretches protected from DNase I digestion by PhaR-His 6 binding are shown in hatched squares. Hocked arrowheads and the labels " +1 " indicate the transcriptional start point (shown in blue letters in the upper strands). The -35 and -10 regions are underlined and the ATG initiating codons are shown in red letters in the upper strands 


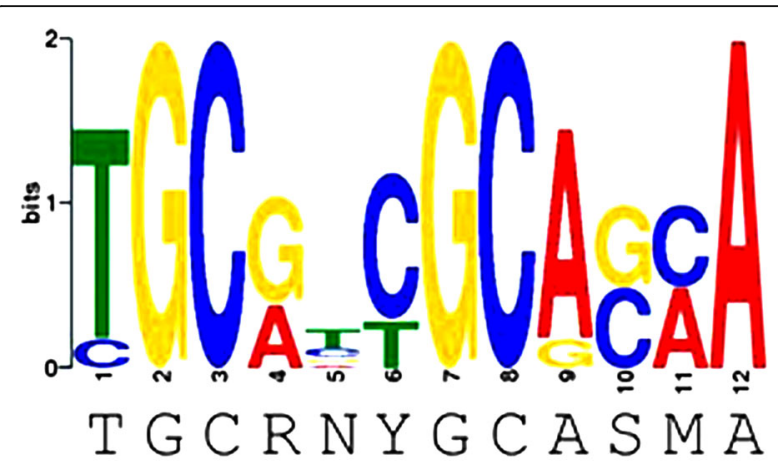

(R: A or G, Y: C or T, S: C or G, M: A or C)

Fig. 5 Prediction of putative PhaR-binding consensus sequence. Nucleotide sequences of the promoter regions of phaP5, phaR, phaZ1, phaZ3, the CyoABCD operon, and the exoZ-containing operon starting with blr2367 were aligned with three 12-mer sequences for PhaR-binding found in the phaP1 and phaP4 promoter regions by GLAM2 in MEME suit under the default settings for analysis to deduce a motif for PhaR binding. The graphical summary of GLAM2 analysis is shown with the consensus sequence TGCRNYGCASMA (R: A or $\mathrm{G}, \mathrm{Y}$ : C or T, S: C or G, M: A or C) at the bottom

in $\Delta \mathrm{phaR}$ and transcription of $p p c$ and $p p s A$ increased, whereas those of $p c k A$ and exaA decreased. Together, these changes may cause a distortion in carbon metabolism. However, amounts of EPS produced in $\triangle$ phaR were approximately double those produced in USDA110 (Fig. 1), indicating that part of the energy and resources for PHB accumulation may be used for EPS production in $\triangle$ phaR. In addition, transcription of the operon containing exoZ increased in $\triangle$ phaR. Reduced EPS production was reported in an exoZ mutant strain of $S$. meliloti [33]. Therefore, the opposite effect may occur upon elevated expression of exoZ in $\triangle$ phaR, resulting in an increase in EPS production. Recently, the similar phenotypic changes were reported for another mutant with phaR inactivation [9]. On the other hand, we found $\Delta \mathrm{phaR}$ improved thermal stress tolerance in both YEM and TY regardless of PHB accumulation (Additional file 2: Figure S1). It is known that PhaP expressed in E. coli exerted a molecular chaperone-like effect [34, 35]; in $\triangle \mathrm{phaR}$, PhaP1, PhaP4, and PhaP5 are produced in excess, which may restore proteins denatured under heat stress conditions. In addition, transcription of both blr2761 and bll6069 was elevated in $\Delta$ phaR, each of which encodes a protein containing the Usp motif, similar to E. coli uspA, which is involved in stress tolerance [31].

Interestingly, $\Delta$ phaR grew better under microaerobic conditions (Additional file 3: Figure S2). In $\Delta$ phaR, some genes that function under hypoxic conditions were enhanced (Additional file 4: Table S2), such as fixNOQP, fixGHIS, napEDABC, and nirK, and were increased, and these genes could be involved in enhancing growth and nitrogen fixation under anaerobic conditions. Nevertheless, the $\Delta$ phaR nodulation in soybean was nearly normal, with no obvious alteration in symbiotic nitrogen fixation under laboratory conditions (data not shown). The cyoABCDE operon of $E$. coli is induced under higher oxygen conditions [36], whereas in $R$. etli the cyoABCD operon was induced under hypoxic conditions to support growth [27]. Therefore, if the cyoABCD operon functions similarly in $B$. diazoefficiens as it does in $R$. etli, the increased expression of the $c y o A B C D$ operon in $\triangle \mathrm{phaR}$ may explain its better growth under the microaerobic conditions.

PhaR could directly repress transcription of various genes; at least 28 of the genes may be negatively regulated and associated with a PhaR-binding site, including the $c y o A B C D$ operon, the operon involved in EPS production that contains exoZ, and $p p c$ (Table 2). Inactivation of phaR also led to downregulation of a large number of genes, suggesting PhaR may function as a positive regulator; at least 42 of the genes may be associated with a PhaRbinding site (Table 3). Some genes involved in carbon metabolism, such as the $p d h A B$ operon, $p c k A$, and exaA could be upregulated through PhaR binding to their promoter regions (Fig. 6). In addition, PhaR binds to the promoter region of phbB2 (Fig. 6), previously shown to be important for PHB synthesis [12], the transcription of which decreased twofold in $\Delta$ phaR (Fig. 2). In $R$. etli, PhaB disappeared in the aniA (a phaR homolog) mutant [18], implying direct involvement of $\mathrm{PhaR}$ in the production of PhbB2. In any case, further studies are required to understand the mechanism of $\mathrm{PhaR}$ in transcriptional activation.

We found two PhaR-His 6 binding sites in the phaP1 promoter and one in the phaP4 promoter, as determined by DNase I footprint analyses (Fig. 4). The gel mobility shift assays indicated a higher PhaR-His ${ }_{6}$ affinity for the phaP1 promoter than for the phaP4 promoter (Fig. 3), suggesting that PhaR could exert tighter control over phaP1 than phaP4. In a previous report, transcriptional activity of the phaR promoter in a phaR mutant doubled over that of the wild-type strain of $B$. diazoefficiens [9]. In the present study, the gel mobility shift assays revealed that $\mathrm{PhaR}-\mathrm{His}_{6}$ binds to the phaR promoter region inefficiently (Fig. 3), implying possible leakiness in its own repression, allowing a constitutive level of PhaR expression in the cell. We demonstrated that DNA binding of PhaR-His ${ }_{6}$ was abolished in the presence of PHB in vitro (Additional file 7: Figure S5). These results were in agreement with the previous finding in Paracoccus denitrificans [37] and our previous observation that PhaR competed for PHB binding with PhaPs and was released from PHB as concentrations of PhaPs increased [12]. The liberated PhaR then binds DNA again to repress the PhaP genes. In our previous studies, PhaP4 showed a higher affinity for PHB than PhaP1 [12]. It is suggested that PhaR could control phaP1 more tightly than phaP4 (Fig. 3). 
Table 2 PhaR-repressed genes associated with a PhaR-binding consensus sequence

\begin{tabular}{|c|c|c|c|c|c|}
\hline Gene or orf & PhaR binding site & USDA110 RPKM & $\Delta$ phaR RPKM & Fold change $^{a}$ & Description \\
\hline cyoA (blr0149) & TGCGGCGCAGCA & 22.29 & 77.7 & 3.49 & cytochrome O ubiquinol oxidase subunit II \\
\hline phaZ3 (blr0899) & TGCAGTGCAGCA & 32.68 & 89.48 & 2.74 & poly(3-hydroxyalkanoate) depolymerase \\
\hline phaZ1 (blr0908) & CGCATCGCAGCA & 129.32 & 542.25 & 4.19 & poly(3-hydroxybutyrate) depolymerase \\
\hline rhIE (bll1447) & TGCAGTGCAGAA & 152.94 & 551.69 & 3.61 & dead-box ATP-dependent RNA helicas \\
\hline blr2242 & TGCGCCGCAGCA & 66.21 & 190.65 & 2.88 & unknown protein \\
\hline bll2363 & TGCGCCGCACAA & 374.44 & 1256.32 & 3.36 & unknown protein \\
\hline blr2367 & TGCACCGCAGCA & 55.14 & 347.34 & 6.30 & acetyltransferase \\
\hline bll2471 & TGGATCGCACCA & 86.78 & 512.16 & 5.90 & $\begin{array}{l}\text { hypothetical protein (Hemerythrin HHE cation } \\
\text { binding region) }\end{array}$ \\
\hline bll2537 & CGCGTCGCAGCA & 39.54 & 83.05 & 2.10 & hypothetical protein \\
\hline phaP5 (blr2887) & TGCATCGCACAA & 122.86 & $15,882.63$ & 129.27 & phasin \\
\hline ppc (blr2955) & TGCGCTGCGGCA & 46.27 & 93.71 & 2.03 & PEP carboxylase \\
\hline bll3794 & TGCATTGCAGCG & 104.59 & 404.72 & 3.87 & hypothetical protein \\
\hline blr4162 & TGCATCGCACCA & 79.46 & 931 & 11.72 & $\begin{array}{l}\text { hypothetical protein (cellulose synthase catalytic } \\
\text { subunit protein) }\end{array}$ \\
\hline bsr4236 & TGCATTGCAACA & 38.73 & 435.02 & 11.23 & unknown protein \\
\hline nifR (blr4486) & TGCAGTGCAGCA & 112.16 & 225.25 & 2.01 & nitrogen regulation protein \\
\hline metN (blr4501) & TGCACCGCAACA & 14.42 & 29.08 & 2.02 & probable $A B C$ transporter ATP-binding protein \\
\hline bsr4726 & TGCGGTGCACAC & 157.38 & 1402.14 & 8.91 & hypothetical protein \\
\hline bll4785 & CGCGCCGCACAA & 90.88 & 279.72 & 3.08 & transcriptional regulatory protein Fis family \\
\hline phaP1 (bll5155) & TGCAACGCACAA & 215.67 & $10,738.12$ & 49.79 & phasin \\
\hline bsr5273 & TGCGGTGCATCA & 45.13 & 222.49 & 4.93 & hypothetical protein \\
\hline bll5524 & AGCAGTGCAGCA & 106.54 & 220.06 & 2.07 & hypothetical protein \\
\hline blr5525 & AGCAGTGCAGCA & 23.4 & 46.85 & 2.00 & hypothetical protein \\
\hline blr5594 & TGCGGCGCACAA & 26.16 & 71.75 & 2.74 & MFS permease \\
\hline bll6290 & TGCGGCGGACCA & 146.99 & 458.11 & 3.12 & two-component response regulator \\
\hline blr6718 & TGCCATGCAGCA & 42.45 & 111.4 & 2.62 & hypothetical protein \\
\hline nirk (blr7089) & TGCGCTGCAACA & 13.49 & 41.36 & 3.07 & respiratory nitrite reductase \\
\hline phap4 (bll7395) & TGCGCTGCACAA & 489.78 & 2956.78 & 6.04 & phasin \\
\hline blr7872 & TGCGCTGCAACA & 6.30 & 20.63 & 3.27 & HlyD family secretion protein \\
\hline
\end{tabular}

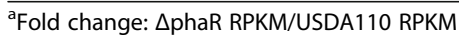

Therefore, given its higher affinity for $\mathrm{PHB}$, PhaP4 may be readily produced to release PhaR from PHB. It is possible that PhaP5 may also be play an important role, as it was induced almost fivefold over phaP4 in $\Delta$ phaR (Table 2). Since PhaP1, PhaP4, and PhaP5 were all overexpressed in $\triangle$ phaR, PHB could be covered immediately by these PhaPs, thereby inhibiting the expansion of PHB granules. In addition, increasing expression of phaZ1 and phaZ3 could result in degradation of PHB. Previous in vitro experiments revealed that PhaPs on the surface of PHB granules could contribute to activity of PhaZ enzymes, and the possible interaction between PhaPs and PhaZ enzymes may have an effect on the decrease of accumulated PHB $[38,39]$.

In Rhodobacter sphaeroides, PhaR repressed transcription of phaZ for PHB degradation [40]. In the present study, we found that transcription of phaZ1 in $\triangle \mathrm{phaR}$ increased about fourfold over that in USDA110 (Table 2) and PhaR$\mathrm{His}_{6}$ bound to the phaZ1 promoter region in vitro (Fig. 3). Furthermore, a third gene for PHB degradation, phaZ3, was found; its expression was elevated by inactivation of phaR (Table 2), and PhaR- $\mathrm{His}_{6}$ binding to its promoter region was demonstrated in vitro (Fig. 3). Therefore, PhaR of $B$. diazoefficiens could negatively and directly control transcription of the two PHB degrading genes, phaZ1 and phaZ3. PhaZ3 has the esterase PHB depolymerase motif, whereas PhaZ1 and PhaZ2 have the PHB depolymerase C-terminus motif (data not shown). In addition, a blast search against the genome of $R$. eutropha strain H16 revealed that PhaZ3 shares a homology to PhaZ7 of H16 (E-value $=5 \mathrm{e}-16)$. It was demonstrated that induction of phaZ7 in a recombinant $E$. coli strain greatly reduced PHB 
Table 3 PhaR-activated genes associated with a PhaR-binding consensus sequence

\begin{tabular}{|c|c|c|c|c|c|}
\hline Genes or orf & PhaR binding site & USDA110 RPKM & $\Delta$ phaR RPKM & Fold change ${ }^{a}$ & Description \\
\hline phbB2 (bll0225) & TGCGCTGCACAC & 154.34 & 74.52 & 2.07 & acetoacetyl CoA reductase \\
\hline $\operatorname{rag} A(b \| 10304)$ & TGCGACGCCGCA & 8.95 & 2.29 & 3.91 & two-component response regulator \\
\hline blr0305 & TGCGACGCCGCA & 45.61 & 16.48 & 2.77 & unknown protein \\
\hline bll0805 & CGCAACGCACAA & 185.17 & 76.29 & 2.43 & hypothetical protein \\
\hline blr0806 & CGCAACGCACAA & 52.09 & 23.58 & 2.21 & hypothetical protein \\
\hline bll1416 & TGCGCGGCAGCA & 52.49 & 20.56 & 2.55 & unknown protein \\
\hline blr2204 & TGCAGTCCAGAA & 19.07 & 9.05 & 2.11 & transcriptional regulatory protein AraC family \\
\hline bll2446 & TTCGCCGCAGAA & 275.72 & 129.25 & 2.13 & hypothetical protein \\
\hline bsr2601 & TGCACCGCAGCC & 32.44 & 15.65 & 2.07 & unknown protein \\
\hline blr2810 & TGCATTGCGCAA & 15.88 & 7.35 & 2.16 & aldo/keto reductase \\
\hline bll2914 & TGCGCTGGAGAA & 25.07 & 9.61 & 2.61 & probable amidase \\
\hline bll3387 & TGCGCCGCAACA & 278.27 & 54.25 & 5.13 & unknown protein \\
\hline blr3795 & TGCATTGCAGCG & 40.09 & 19.03 & 2.11 & $\mathrm{ABC}$ transporter HlyB/MsbA family \\
\hline blr3904 & TGCAGTGCTGCA & 312.33 & 109.92 & 2.84 & probable iron transport protein \\
\hline blr4188 & CGCAGTGCAGCA & 28.85 & 12.98 & 2.22 & hypothetical protein \\
\hline bll4430 & TGCAGCGCAGCA & 104.34 & 38.21 & 2.73 & hypothetical protein \\
\hline bsr4431 & TGCAGCGCAGCA & 216.36 & 77.85 & 2.78 & hypothetical protein \\
\hline pdhA (bll4783) & TGCAGTGCGGCA & 249.67 & 111.10 & 2.25 & pyruvate dehydrogenase alpha subunit \\
\hline bll4833 & TGCGGCGCACCA & 530.73 & 140.09 & 3.79 & unknown protein \\
\hline bsr4834 & TGCGGCGCACCA & 168.95 & 52.96 & 3.19 & unknown protein \\
\hline blr4841 & TGCCGCGCACAA & 30.64 & 14.59 & 2.10 & unknown protein \\
\hline bll4952 & TGCATCGCACAA & 27.07 & 7.50 & 3.61 & NfeD protein homolog \\
\hline bll5160 & TGCGGCGCACAA & 106.54 & 46.40 & 2.30 & conserved hypothetical protein; putative alpha/beta-Hydrolases \\
\hline bsl5321 & CGCGGCGCAGCA & 415.34 & 205.51 & 2.02 & unknown protein \\
\hline bll5335 & TGCGCCGGACAA & 35.29 & 16.85 & 2.09 & putative thiolase \\
\hline blr5540 & TGCGGTGCCCAA & 231.26 & 58.07 & 3.98 & hypothetical protein \\
\hline bsl5717 & TGCGGCGCCCAA & 109.78 & 34.94 & 3.14 & hypothetical protein \\
\hline bll5961 & TGCGGTGCAACA & 12.66 & 4.23 & 2.99 & transcriptional regulatory protein Crp family \\
\hline blr5962 & TGCGGTGCAACA & 40.98 & 7.40 & 5.54 & ABC transporter ATP-binding protein \\
\hline bll6121 & TGCAGCGCACAA & 21.88 & 9.39 & 2.33 & probable sulfite oxidase \\
\hline bll6206 & CGCGCCGCACAA & 74.19 & 35.28 & 2.10 & hypothetical protein \\
\hline exaA (blr6207) & CGCGCCGCACAA & 93.32 & 23.85 & 3.91 & probable quinoprotein ethanol dehydrogenase precursor \\
\hline blr6443 & TGCAATGCAACA & 13.79 & 2.83 & 4.87 & $A B C$ transporter permease protein \\
\hline blr6465 & CGCGATGCACAA & 39.03 & 14.29 & 2.73 & putative steroid monooxygenase \\
\hline bll6733 & TGCGACGAAGCA & 45.09 & 21.88 & 2.06 & putative amidase \\
\hline pqqA (bsr6735) & TGCAGTGCAACA & 156.84 & 40.03 & 3.92 & putative pyrroloquinoline quinone synthesis protein $\mathrm{A}$ \\
\hline blr6837 & CGCAGTGCAGCA & 34.10 & 7.30 & 4.67 & hypothetical protein \\
\hline blr6886 & CGCATTGCACAA & 57.55 & 25.47 & 2.26 & transcriptional regulatory protein MarR family \\
\hline bll7487 & TGCGGAGCACAA & 456.33 & 176.20 & 2.59 & unknown protein \\
\hline bll7511 & TGCAACGCAGAT & 167.13 & 62.44 & 2.68 & unknown protein \\
\hline bll7663 & TGCACCGCAGCA & 57.90 & 24.14 & 2.40 & unknown protein \\
\hline pckA (b\|ll8141) & TGCGACGCACAA & 386.03 & 107.43 & 3.59 & phosphoenolpyruvate carboxykinase \\
\hline
\end{tabular}

${ }^{a}$ Fold change: USDA110 RPKM/AphaR RPKM 


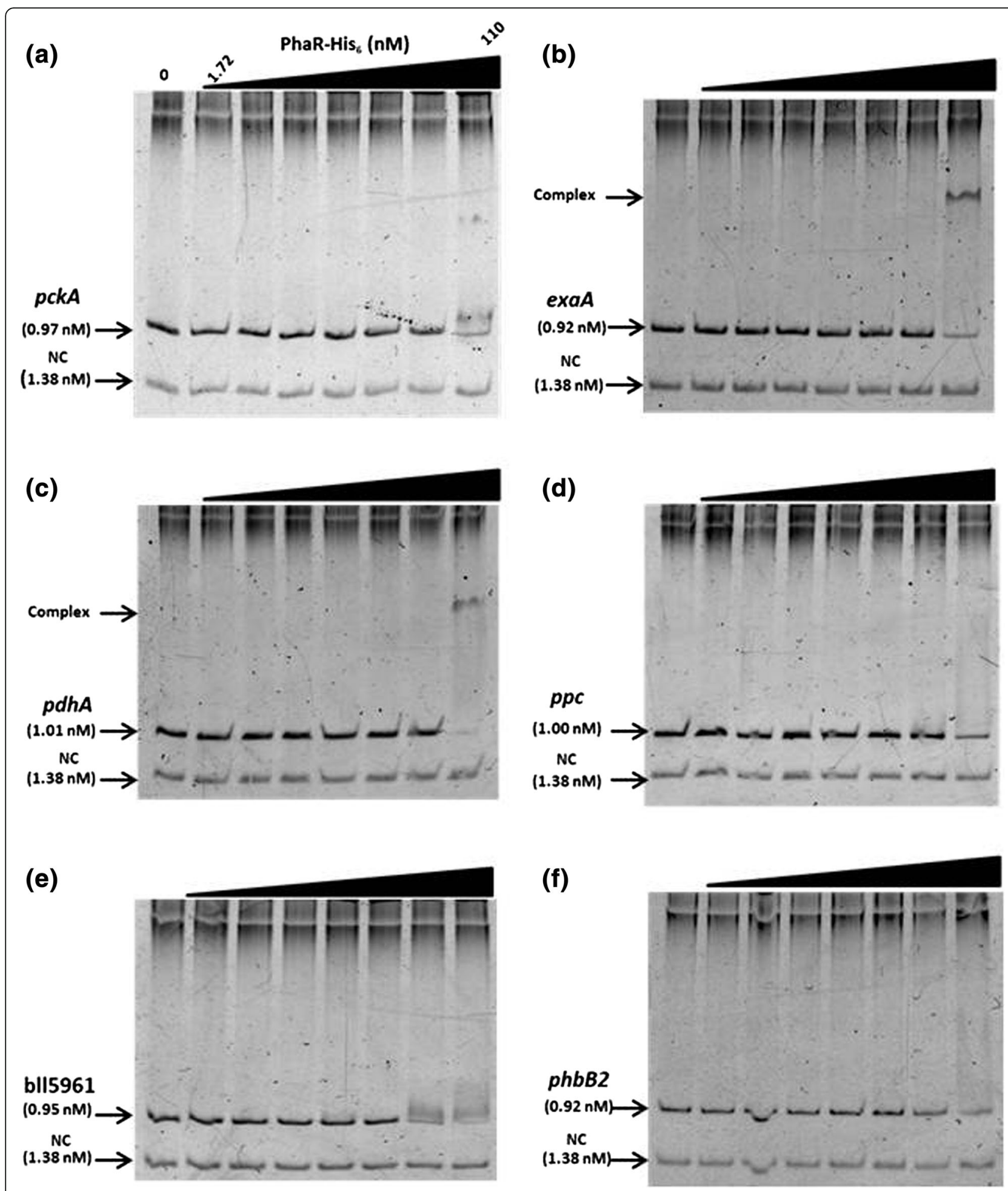

Fig. 6 PhaR binding to DNA fragments of the promoter regions of pckA (bll8141) (a), exaA (blr6207) (b), pdhAB (bll4783-bll4779) operon (c), ppc (blr2955) (d), bll5961-blr5962 operon (e), and phbB (bll0225) (f). Experimental conditions and lane assignments are the same as shown in (Fig. 3) 
accumulation [41]. Therefore, PhaZ3 may play an important role in PHB degradation in B. diazoefficiens.

The transcriptome analysis revealed an additional PhaP gene, phaP5, the expression of which was prominent in $\triangle \mathrm{phaR}$ compared with USDA110 (Additional file 4: Tables 2). PhaP5 possesses the phasin 2 motif, similar to that found in the other four PhaPs. In the present study, we demonstrated $\mathrm{PhaR}-\mathrm{His}_{6}$ binding to the promoter regions of these PhaP genes using gel mobility shift assays (Fig. 3). It is likely that PhaR binds to the promoter regions of phaP1, phaP4, and phaP5 and represses their transcription under non-PHB-accumulating conditions. These results suggested that PhaP5 may play an important role in PHB accumulation together with PhaP1 and PhaP4.

It was also revealed that some of the genes regulated by FixK $\mathrm{K}_{2}$, a global transcriptional activator for adaptation to microaerobic conditions, increased their expression in $\triangle$ phaR, PhaR-His ${ }_{6}$ may bind to the fixK2 promoter region. However, a DNA fragment of the fixK2 promoter region exhibited no interaction with $\mathrm{PhaR}-\mathrm{His}_{6}$ under the conditions used in the present study (data not shown). When cultured under microaerobic conditions, transcription of fixK2 increased in a mutant strain of $B$. diazoefficiens lacking phaR [9]. In the present study, however, transcription of fixK2 in $\triangle$ phaR was not elevated significantly (Additional file 4: Table S2), and no PhaR-binding site was predicted in the promoter region of fixK2. Nevertheless, FixK $K_{2}$ is activated in response to a moderate decrease in oxygen concentration [28]. As described above, we found that transcription of both the napEDABC operon and nirK, which function in the uptake and reduction of nitric acid under anaerobic conditions, was elevated in $\Delta$ phaR $[29,30]$. This implies that phaR inactivation may make the intracellular environment less aerobic as the metabolic circuit is modulated to accelerate respiration to consume more oxygen, and thus available oxygen could decrease to activate $\mathrm{FixK}_{2}$ for induction of its targets. PhbC3 could be active as a homodimer catalyzing $\mathrm{PHB}$ polymerization, whereas $\mathrm{PhbC5}$ may bind to $\mathrm{PhbC} 3$ to form an inactive heterodimer [9]. In the present study, we found that PhaR may not directly control $p h b C 3$ and $p h b C 5$. It was previously suggested that $\mathrm{FixK}_{2}$ may be involved in the induction of phbC5 [9]. Expression of phbC5 in $\triangle \mathrm{phaR}$ appeared to increase slightly (Fig. 2), which may be one of the additional reasons why PHB did not accumulate in $\Delta$ phaR (Fig. 1).

\section{Conclusions}

PhaR of B. diazoefficiens USDA110 is a DNA-binding transcription factor that is originally known to control the PHB granule stabilization in response to the intracellular levels of PHB. It was found that inactivation of phaR in USDA110 led to pleiotropic changes in cellular processes not only in PHB accumulation. It was demonstrated that
PhaR regulated transcription of its various target genes, binding to their promoter regions that contain the relaxed consensus sequence, TGCRNYGCASMA (R: A or G, Y: C or T, S: C or G, M: A or C). These results suggest that PhaR could regulate a large number of genes to coordinate metabolism holistically in response to PHB accumulation.

\section{Additional files}

\begin{abstract}
Additional file 1: Table S1. Oligonucleotides used in this study. Names of oligonucleotides and their nucleotide sequences are listed. (PDF $66 \mathrm{~kb}$ ) Additional file 2: Figure S1. Heat stress resistance of $B$. diazoefficiens. $B$. diazoefficiens strains USDA110 (WT) and $\Delta$ phaR were pre-cultured in TY (a) and in YEM (b) for 2 and 12 days, respectively, and then diluted to yield $\mathrm{OD} 600=0.1$ in respective fresh media. For the heat shock stress experiments, the diluted cultures were incubated with shaking at $160 \mathrm{rpm}$ at $50{ }^{\circ} \mathrm{C}$ for 5 and $10 \mathrm{~min}$, respectively. After incubation, the cultures were serially diluted 1:10 for 5 times. Each of the diluted aliquots $(5 \mu \mathrm{L})$ were spotted from left to right on to PSY plates and incubated at $28^{\circ} \mathrm{C}$ for 1 week for colony formation. (PDF $169 \mathrm{~kb}$ )
\end{abstract}

Additional file 3: Figure S2. Colony formation of $B$. diazoefficiens under aerobic (a) and microaerobic (b) conditions. Cultures of $B$. diazoefficiens strains USDA110 (WT) and $\Delta$ phaR $(O D 600=0.1)$ were serially diluted 10 times, spotted on to TY plates, and grown under aerobic (a) and anaerobic (b) conditions at $28^{\circ} \mathrm{C}$. Pictures were taken after 5 (a) and 19 (b) days of incubation. (PDF $340 \mathrm{~kb}$ )

Additional file 4: Table S2. Summary of RNA sequencing analysis to compare transcriptome of USDA110 and $\Delta$ phaR. This is an excel spreadsheet summarizing the results of RNA sequencing analysis. (XLSX 1269 kb)

Additional file 5: Figure S3. Overview of RNA sequencing analysis data quality. (a) Comparison of USDA110 (WT) and $\triangle$ phaR RPKM values. (b) Comparison of log2 transformed data of quantitative RT-PCR and RNA sequencing. Fold change refers to the relative expression in $\Delta$ phaR to USDA110 (WT). (PDF 146 kb)

Additional file 6: Figure S4. Purification of PhaR-His 6 . PhaR-His 6 was purified by Ni-Co affinity chromatography and its purity analyzed using a 12\% SDS-polyacrylamide gel. Lane $\mathrm{M}$, molecular weight markers; lane 1, flow through fraction; lane 2 , wash fraction; lanes $3-5$, eluted fractions with 40, 100, and $200 \mathrm{mM}$ imidazole, respectively. The purified PhaR-His 6 is found at approximately $22.6 \mathrm{kDa}$. (PDF $128 \mathrm{~kb}$ )

Additional file 7: Figure S5. PhaR binding to DNA fragments containing the promoter regions of phaP paralogs in the presence of PHB. A fixed amount of DNA fragments containing the phaP1 (a) and phaP4 (b) promoter regions were incubated with the indicated amount of $\mathrm{PhaR}^{-H \mathrm{H}_{6}}$ and various amounts of PHB (0 to $50 \mathrm{ng}$ per reaction as indicated). The control lane contained neither PhaR-His 6 nor PHB. The negative control (NC) DNA is the same as Fig. 3. (PDF $63 \mathrm{~kb}$ )

\section{Abbreviations}

EPS: Exopolysaccharides; Km: Kanamycin; LB: Lysogeny broth medium; PHA: Polyhydroxyalkanoate; PhaP: Phasin protein; PHB: Poly-3hydroxybutyrate; Pm: Polymyxin B; PSY: Peptone salts yeast extract medium; YEM: Yeast extract mannitol medium

\section{Acknowledgements}

The authors would like to thank Enago (http://www.enago.jp) for the English language review.

\section{Funding}

This work was financially supported by the Ministry of Education, Culture, Sports, Science and Technology (MEXT), Japan, under Special Coordination Funds for Promoting Science and Technology, Creation of Innovative Centers for Advanced Interdisciplinary Research Areas, and KAKENHI 17 K19237 and $18 \mathrm{H} 02128$ to $\mathrm{KY}$. 


\section{Availability of data and materials}

The transcriptome analysis data are available in the DDBJ Sequence Read Archive (DRA) database under accession number DRA005621. The other data and materials obtained during the current study are available from the corresponding author on reasonable request.

\section{Authors' contributions}

$\mathrm{KY}$ conceived and conducted the project. SN performed most of the experiments, including mutant construction, phenotypic tests, and analyses of transcriptional and DNA-binding function of PhaR, with the indispensable assistance of TK. KT, SI, and ST helped SN for transcriptome analysis, phenotypic data analysis, and protein works, respectively. KY and CK wrote the final manuscript together. All the authors have agreed with this publication.

\section{Ethics approval and consent to participate}

Not applicable.

\section{Consent for publication}

Not applicable.

\section{Competing interests}

The authors declare that they have no competing interests.

\section{Publisher's Note}

Springer Nature remains neutral with regard to jurisdictional claims in published maps and institutional affiliations.

\section{Author details}

'Department of Agrobioscience, Kobe University, 1-1 Rokkodai, Nada, Kobe 657 8501, Japan. ${ }^{2}$ Department of Science, Technology and Innovation, Kobe University, 1-1 Rokkodai, Nada, Kobe 657 8501, Japan. ${ }^{3}$ Department of Biological Science, California State University, Stanislaus, Turlock, CA 95382, USA.

\section{Received: 6 June 2018 Accepted: 15 October 2018}

\section{Published online: 24 October 2018}

\section{References}

1. Anderson AJ, Dawes EA. Occurrence, metabolism, metabolic role, and industrial uses of bacterial polyhydroxyalkanoates. Microbiol Rev. 1990;54: 450-72.

2. Tokiwa Y, Calabia BP, Ugwu CU, Aiba S. Biodegradability of plastics. Int J Mol Sci. 2009:10:3722-42

3. Bashan Y, Holguin G, de-Bashan LE. Azospirillum-plant relationships: physiological, moecular, agricultural, and environmental advances (19972003). Can J Microbiol. 2004;50:521-77.

4. Kadouri $D$, Jurkevitch $E$, Okon $Y$. Involvement of the reserve material poly- $\beta$ hydroxybutyrate in Azospirillum brasilense stress endurance and root colonization. Appl Environ Microbiol. 2003;69:3244-50.

5. Koskimäki JJ, Kajula M, Hokkanen J, Ihantola EL, Kim JH, Hautajärvi H, et al. Methyl-esterified 3-hydroxybutyrate oligomers protect bacteria from hydroxyl radicals. Nat Chem Biol. 2016;12:332-8.

6. Ratcliff WC, Kadam SV, Denison RF. Poly-3-hydroxybutyrate (PHB) supports survival and reproduction in starving rhizobia. FEMS Microbiol Ecol. 2008;65: 391-9.

7. Wang C, Sheng X, Equi RC, Trainer MA, Charles TC, Sobral BW. Influence of the poly-3-hydroxybutyrate (PHB) granule-associated proteins (PhaP1 and PhaP2) on PHB accumulation and symbiotic nitrogen fixation in Sinorhizobium meliloti Rm1021. J Bacteriol. 2007;189:9050-6.

8. Bergensen FJ, Peoples MB, Turner GL. A role for poly- $\beta$-hydroxybutyrate in bacteroids of soybean root nodules. Proc R Soc Lond B Biol Sci. 1991;245: 59-64.

9. Quelas JI, Mesa S, Mongiardini EJ, Jendrossek D, Lodeiro AR. Regulation of polyhydroxybutyrate synthesis in the soil bacterium Bradyrhizobium diazoefficiens. Appl Environ Microbiol. 2016:82:4299-308.

10. Trainer MA, Charles TC. The role of PHB metabolism in the symbiosis of rhizobia with legumes. Appl Environ Microbiol. 2006;71:377-86.

11. Lodwig EM, Leonard M, Marroqui S, Wheeler TR, Findlay K, Downie JA, et al. Role of polyhydroxybutyrate and glycogen as carbon storage compounds in pea and bean bacteroids. Mol Plant-Microbe Interact. 2005;18:67-74.
12. Yoshida K, Takemoto Y, Sotsuka T, Tanaka K, Takenaka S. PhaP phasins play a principal role in poly- $\beta$-hydroxybutyrate accumulation in free-living Bradyrhizobium japonicum. BMC Microbiol. 2013;13:290.

13. Pötter M, Müller $H$, Steinbüchel A. Influence of homologous phasins (PhaP) on PHA accumulation and regulation of their expression by the transcriptional repressor PhaR in Ralstonia eutropha H16. Microbiology. 2005;151:825-33.

14. York GM, Stubbe J, Sinskey AJ. The Ralstonia eutropha PhaR protein couples synthesis of the PhaP phasin to the presence of polyhydroxybutyrate in cells and promotes polyhydroxybutyrate production. J Bacteriol. 2002;184: 59-66.

15. Quelas Jl, Mongiardini EJ, Pérez-Giménez J, Parisi G, Lodeiro AR. Analysis of two polyhydroxyalkanoate synthases in Bradyrhizobium japonicum USDA 110. J Bacteriol. 2013;195:3145-55.

16. Kuchta K, Chi L, Fuchs H, Pötter M, Steinbüchel A. Studies on the influence of phasins on accumulation and degradation of PHB and nanostructure of PHB granules in ralstonia eutropha H16. Biomacromolecules. 2007;8:657-62.

17. Korotkova N, Chistoserdova L, Lidstrom ME. Poly- $\beta$-hydroxybutyrate biosynthesis in the facultative methylotroph Methylobacterium extorquens AM1: identification and mutation of gap11, gap20, and phaR. J Bacteriol. 2002;184:6174-81.

18. Povolo S, Casella S. A critical role for aniA in energy-carbon flux and symbiotic nitrogen fixation in Sinorhizobium meliloti. Arch Microbiol. 2000;174:42-9.

19. Encarnación S, del Carmen Vargas M, Dunn MF, Dávalos A, Mendoza G, Mora $Y$, et al. AniA regulates reserve polymer accumulation and global protein expression in Rhizobium etli. J Bacteriol. 2002;184:2287-95.

20. Bertani G. Studies on lysogenesis. I. the mode of phage liberation by lysogenic Escherichia coli. J Bacteriol. 1951;62:293-300.

21. Regensburger $B$, Hennecke $H$. RNA polymerase from Rhizobium japonicum. Arch Microbiol. 1983;135:103-9.

22. Vincent JM. A manual for the practical study of root nodule bacteria. International biological Programme. Oxford: Blackwell Scientific; 1970.

23. Tombolini R, Povolo S, Buson A, Squartini A, Nuti MP. Poly- $\beta$ hydroxybutyrate (PHB) biosynthetic genes in Rhizobium meliloti 41. Microbiology. 1995;141:2553-9.

24. Law JH, Slepecky RA. Assay of poly- $\beta$-hydroxybutyric acid. J Bacteriol. 1960;82:33-6.

25. Quelas Jl, Mongiardini EJ, Casabuono A, López-García SL, Althabegoiti MJ, Covelli JM, et al. Lack of galactose or galacturonic acid in Bradyrhizobium japonicum USDA 110 exopolysaccharide leads to different symbiotic responses in soybean. Mol Plant-Microbe Interact. 2010;23:1592-604.

26. Chun Y, Yin ZD. Glycogen assay for diagnosis of female genital Chlamydia trachomatis infection. J Clin Microbiol. 1998;36:1081-2.

27. Lunak ZR, Noel KD. A quinol oxidase, encoded by $c y o A B C D$, is utilized to adapt to lower $\mathrm{O}_{2}$ concentrations in Rhizobium etli CFN42. Microbiology. 2015:161:203-12.

28. Mesa S, Hauser F, Friberg M, Malaguti E, Fischer HM, Hennecke H. Comprehensive assessment of the regulons controlled by the FixLJ-FixK2FixK1 cascade in Bradyrhizobium japonicum. J Bacteriol. 2008;190:6568-79.

29. Bedmar EJ, Robles EF, Delgado MJ. The complete denitrification pathway of the symbiotic, nitrogen-fixing bacterium Bradyrhizobium japonicum. Biochem Soc Trans. 2005:33:141-4.

30. Torres MJ, Bueno E, Mesa S, Bedmar EJ, Delgado MJ. Emerging complexity in the denitrification regulatory network of Bradyrhizobium japonicum. Biochem Soc Trans. 2011;39:284-8.

31. Nyström T, Neidhardt FC. Expression and role of the universal stress protein, UspA, of Escherichia coli during growth arrest. Mol Microbiol. 1994;1 1:537-44.

32. Čuklina J, Hahn J, Imakaev M, Omasits U, Förstner KU, Ljubimov N, et al. Genome-wide transcription start site mapping of Bradyrhizobium japonicum grown free-living or in symbiosis - a rich resource to identify new transcripts, proteins and to study gene regulation. BMC Genomics. 2016;17:302.

33. Buendia AM, Enenkel B, Köplin R, Niehaus K, Arnold W, Pühler A. The Rhizobium meliloti exoZl exoB fragment of megaplasmid 2: ExoB functions as a UDP-glucose 4-epimerase and ExoZ shows homology to NodX of Rhizobium leguminosarum biovar viciae strain TOM. Mol Microbiol. 1991;5: 1519-30.

34. de Almeida A, Catone MV, Rhodius VA, Gross CA, Pettinari MJ. Unexpected stress-reducing effect of PhaP, a poly (3-hydroxybutyrate) granule-associated protein, in Escherichia coli. Appl Environ Microbiol. 2011;77:6622-9. 
35. Mezzina MP, Wetzler DE, de Almeida A, Dinjaski N, Prieto MA, Pettinari MJ. A phasin with extra talents: a polyhydroxyalkanoate granule-associated protein has chaperone activity. Environ Microbiol. 2015;17:1765-76.

36. Cotter PA, Chepuri V, Gennis RB, Gunsalus RP. Cytochrome o (cyoABCDE) and $d(c y d A B)$ oxidase gene expression in Escherichia coli is regulated by oxygen, pH, and the fnr gene product. J Bacteriol. 1990;172:6333-8.

37. Maehara A, Taguchi S, Nishiyama T, Yamane T, Doi Y. A repressor protein, PhaR, regulates polyhydroxyalkanoate (PHA) synthesis via its direct interaction with PHA. J Bacteriol. 2002;184:3992-4002.

38. Handrick R, Technow U, Reichart T, Reinhardt S, Sander T, Jendrossek D. The activator of the Rhodospirillum rubrum PHB depolymerase is a polypeptide that is extremely resistant to high temperature (121 C) and other physical or chemical stresses. FEMS Microbiol Lett. 2004;230:265-74.

39. Handrick R, Reinhardt S, Kimmig P, Jendrossek D. The "intracellular" poly (3hydroxybutyrate) (PHB) depolymerase of Rhodospirillum rubrum is a periplasm-located protein with specificity for native PHB and with structural similarity to extracellular PHB depolymerases. J Bacteriol. 2004;186:7243-53.

40. Chou ME, Chang WT, Chang YC, Yang MK. Expression of four pha genes involved in poly- $\beta$-hydroxybutyrate production and accumulation in Rhodobacter sphaeroides FJ1. Mol Gen Genomics. 2009;282:97-106.

41. Eggers J, Steinbüchel A. Impact of Ralstonia eutropha's poly (3Hydroxybutyrate) (PHB) depolymerases and phasins on PHB storage in recombinant Escherichia coli. Appl Environ Microbiol. 2014;80:7702-9.

42. Kaneko T, Nakamura Y, Sato S, Minamisawa K, Uchiumi T, Sasamoto S, et al. Complete genomic sequence of nitrogen-fixing symbiotic bacterium Bradyrhizobium japonicum USDA110. DNA Res. 2002:9:189-97.

43. Parke D. Construction of mobilizable vectors derived from plasmids RP4, pUC18 and pUC19. Gene. 1990;93:135-7.

44. Schäfer A, Tauch A, Jäger W, Kalinowski J, Thierbach G, Pühler A. Small mobilizable multi-purpose cloning vectors derived from the Escherichia coli plasmids pK18 and pK19: selection of defined deletions in the chromosome of Corynebacterium glutamicum. Gene. 1994;145:69-73.

Ready to submit your research? Choose BMC and benefit from:

- fast, convenient online submission

- thorough peer review by experienced researchers in your field

- rapid publication on acceptance

- support for research data, including large and complex data types

- gold Open Access which fosters wider collaboration and increased citations

- maximum visibility for your research: over $100 \mathrm{M}$ website views per year

At $\mathrm{BMC}$, research is always in progress.

Learn more biomedcentral.com/submissions 